\title{
Self-dual spherical grids
}

\author{
Jack E. Graver \\ Department of Mathematics \\ Syracuse University \\ Syracuse, NY 13244-1150, U.S.A. \\ jegraver@syr.edu
}

\author{
Elizabeth J. Hartung \\ Department of Mathematics \\ Massachusetts College of Liberal Arts \\ North Adams, MA 01247, U.S.A. \\ lizhartung@gmail.com
}

Submitted: Jun 26, 2013; Accepted: Feb 14, 2014; Published: Feb 21, 2014

Mathematics Subject Classifications: 05C10

\begin{abstract}
Self-dual plane graphs have been studied extensively. C. A. B Smith and W. T. Tutte published A class of self-dual maps in 1950 [9]; in 1992, Archdeacon and Richter [1] described a method for constructing all self-dual plane graphs and a second construction was produced by Servatius and Christopher [5] in 1992. Both constructions are inductive. In this paper, we produce four templates from which all self-dual plane graphs with maximum degree 4 (self-dual spherical grids) can be constructed. The self-dual spherical grids are further subdivided into 27 basic automorphism classes. Self-dual spherical grids in the same automorphism class have similar architecture. A smallest example of each class is constructed.
\end{abstract}

\section{Introduction}

The literature on constructing and classifying self-dual plane graphs is rather extensive. In addition to the papers mentioned in the abstract, there are several other relevant papers. The paper by Servatius and Servatius [8] uses matroid methods to construct all self-dual graphs. Other related constructions can be found in [2] by Brinkmann et al., and in [4] by Dutour and Deza. The papers of Servatius and Servatius, [6] and [7], use the symmetry structure to classify self-dual graphs. The feature that distinguishes our approach from these constructions is the set of four templates: each self-dual plane graph with maximum degree 4 is constructed directly by choosing the values for the parameters in the appropriate template. Furthermore one may specify the symmetry structure in the selection of template and parameter values. 


\section{Preliminaries}

By a spherical grid, or an $S G$, we shall mean a plane graph $\Gamma=(V, E, F)$ with vertex and face degrees restricted to 3 and 4 .

Lemma 1. Let $\Gamma$ be a spherical grid with $v_{3}$ vertices of degree-3 and $f_{3}$ faces of degree 3 . Then $v_{3}$ and $f_{3}$ are even numbers and $v_{3}+f_{3}=8$.

Proof. Let $v_{4}$ denote the number of vertices of degree 4 in $\Gamma$, let $f_{4}$ denote the number of faces of degree 4 and let $e$ denote the number of edges. Then:

i. $3 v_{3}+4 v_{4}=2 e$;

ii. $3 f_{3}+4 f_{4}=2 e$;

iii. $\left(v_{3}+v_{4}\right)-e+\left(f_{3}+f_{4}\right)=2$.

Equalities (i) and (ii) are obtained by summing the vertex and face degrees; the third equation is Euler's formula for plane graphs. To maintain parity in the first two equations, $v_{3}$ and $f_{3}$ must be even numbers. Subtracting the first and second equations from 4 times the third gives $v_{3}+f_{3}=8$.

The proof of the next lemma is also straightforward and left to the reader.

Lemma 2. The dual of a spherical grid is another spherical grid.

We let $\Lambda$ denote the infinite plane graph of the tessellation of the plane by squares. One of the easiest ways to describe a spherical grid is to construct a template for it in $\Lambda$. We illustrate this starting with the template in the left-hand diagram of Figure 1. To construct the SG, cut out the left-hand polygon with vertices; fold along the vertical
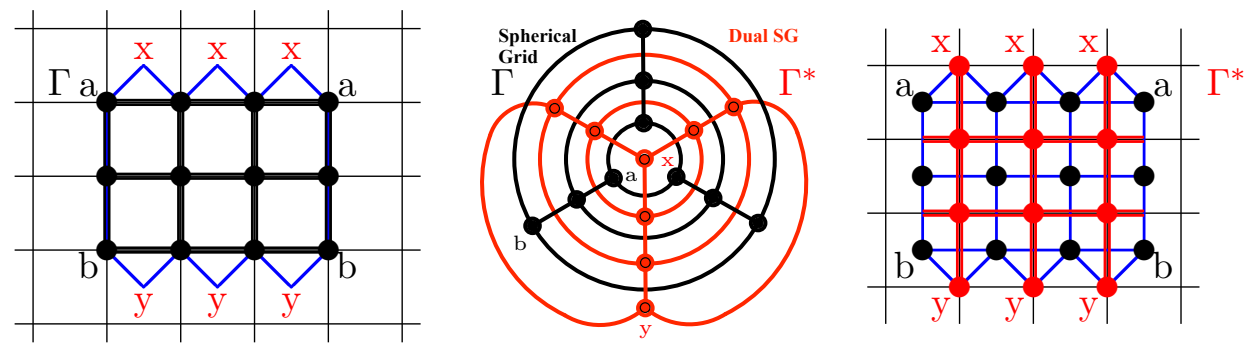

Figure 1: On the left is a template for the black spherical grid in the center. It has $v_{3}=6$, $v_{4}=3, f_{3}=2$ and $f_{4}=6$. Shifting this diagram in $\Lambda$ by $\frac{1}{2}$ unit up and to the right gives a template (pictured on the right) for its dual (red) spherical grid.

segments and identify the two $a-b$ segments to form a triangular tube. Then folding down $x$-tabs forms a triangular "face" at the top; the $y$-tabs give a triangular "face" at the bottom. One easily checks that this is a model of the black SG, $\Gamma$, pictured in the center 
of this figure. If we overlay the grid with the dual grid (in red), we have a template for the dual SG: folding the right-hand template into a polyhedron yields the original spherical grid in black overlaid by its dual spherical grid in red.

A spherical grid is said to be a triangle free spherical grid or a TFSG if it has eight degree-3 vertices and no triangular faces. With the following construction, we may associate a TFSG with any spherical grid $\Gamma$. Draw the dual spherical grid $\Gamma^{*}$ (in red) superimposed on the original spherical grid $\Gamma$ (in black). Bisect each edge in the spherical grid by its dual adding, as a vertex, the intersection of the crossing edges. In Figure 2, we illustrate this construction with the SG from Figure 1. We easily see that each triangular face is replaced by three quadrilateral faces surrounding a (dual) vertex of degree-3 and each quadrilateral face is replaced by four quadrilateral faces surrounding a (dual) vertex of degree 4. We also note that the new vertices, the intersections of edges and dual edges, all have degree 4 . We call these the mixed vertices and color them white. Hence the resulting graph is a TFSG with all eight of its degree-3 vertices among the vertices colored red and black. The nice feature is that this process is reversible.

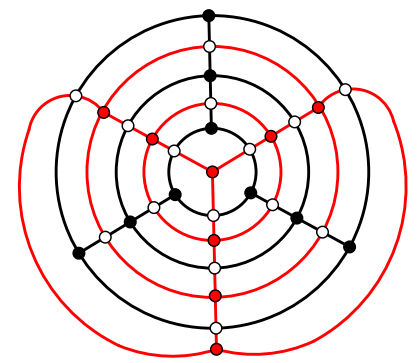

Figure 2: The construction of the triangle free spherical grid (TFSG) for the spherical grid (SG) in Figure 1.

Let $\Gamma=(V, E, F)$ be any TFSG. Observe that, since each face is a quadrilateral, the graph is bipartite. If the eight degree-3 vertices do not belong to the same cell of the bipartition, $\Gamma$ did not come from a spherical grid by the above construction. If all eight degree-3 vertices do belong to the same cell of the bipartition, it could be constructed from a SG and we are able to reverse the construction. Assume that $\Gamma=(V, E, F)$ is a TFSG with all degree-3 vertices in the same class of its bipartition (see the first diagram in Figure 3). Color the vertices in the cell devoid of degree-3 vertices white and, for now, color the remaining vertices green (see the second diagram in Figure 3). We must now partition these green vertices into two cells, red vertices and black vertices. To accomplish this we construct an auxiliary graph. Each face of $\Gamma$ is a square with the green and white vertices alternating around its boundary. We add a diagonal edge in each face joining the two green vertices in its boundary (see the second diagram in Figure 3).

Our auxiliary graph $\Omega$ will have as its vertex set the green vertices and as its edge set these new diagonal edges. Since $\Gamma$ plus diagonals is planar, $\Omega$ is planar. Furthermore, each face of $\Omega$ is a square with a white vertex in its center. It follows that $\Omega$ is bipartite. We arbitrarily color the vertices in one cell red and the vertices in the other cell black (see the third diagram in Figure 3). Since the black and red vertices alternate around a face 


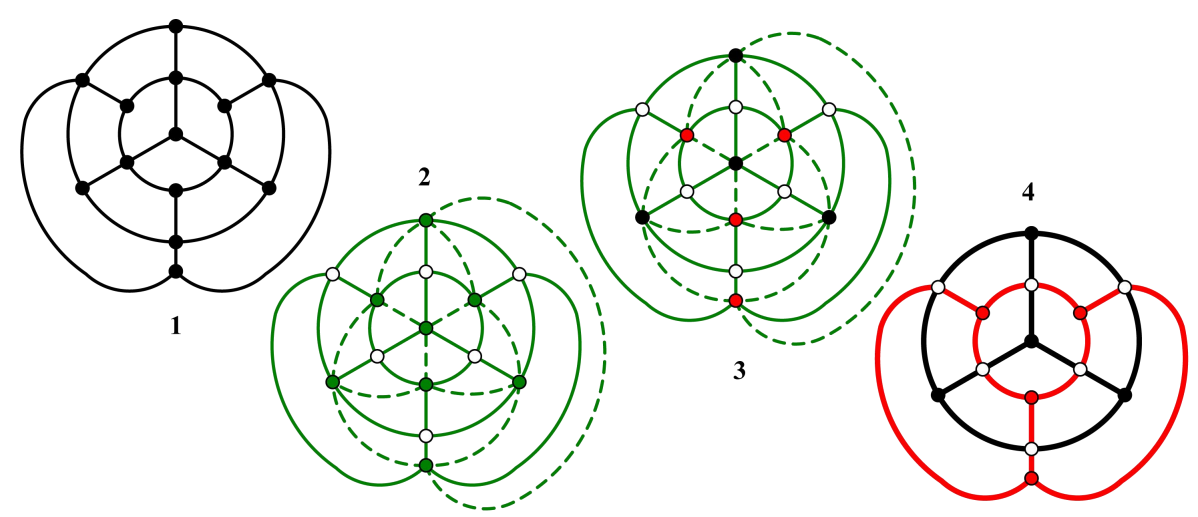

Figure 3: The construction of a dual pair of spherical grids from a triangle free spherical grid.

of $\Omega$, the neighbors of each white vertex in $\Gamma$ alternate red and black around that vertex. Hence in $\Gamma$, each of its edges has one endpoint colored white while the other endpoint is colored either red or black. An edge of $\Gamma$ is now colored red if it has a red endpoint and black if it has a black endpoint (see the last diagram in Figure 3). A TFSG with such a vertex and edge coloring is called a properly colored TFSG.

Finally, let $\Gamma$ be a properly colored TFSG and consider the subgraph of black and white vertices and black edges. All white vertices have degree 2 and are the central vertex of a path of length 2 joining two black vertices. Replacing these paths by single edges results in (the black) spherical grid which we denote by $\Gamma_{b}$. Each face has a single red vertex in its center and the face is square if the red vertex has degree 4 and triangular if the red vertex has degree 3 . Hence, the similarly constructed red SG, $\Gamma_{r}$, is the dual of $\Gamma_{b}$ and $\Gamma$ is the TFSG corresponding to either the black or red SG.

All of our spherical grids are plane graphs. We call an automorphism of a plane graph a symmetry. Almost all of the SGs we consider will be 3-connected and hence all graph automorphisms will be symmetries. However, a few small SGs will not be 3-connected and the distinction between automorphism and symmetry will be necessary. Let $\Gamma$ be a properly colored TFSG. Since $\Gamma$ is a connected bipartite graph, any symmetry of $\Gamma$ must preserve or interchange cells of the bipartition. The white vertices form one partition class and the union of the red and black vertices the other. Since there are no white vertices of degree-3, all symmetries must map the class of white vertices onto itself. Any symmetry of $\Gamma$ induces a symmetry on the auxiliary graph $\Omega$ and, since $\Omega$ is connected and bipartite, that symmetry must either preserve color (map black vertices to black vertices and red to red) or reverse the colors. Hence, a symmetry of $\Gamma$ either induces a symmetry on both $\Gamma_{b}$ and $\Gamma_{r}$ or a graph isomorphism between $\Gamma_{b}$ and $\Gamma_{r}$.

Lemma 3. Let $\Gamma$ be the TFSG constructed from the $S G \Delta$. Then $\Delta$ is self-dual if and only if $\Gamma$ admits a color-reversing symmetry.

Proof. We may assume that $\Delta=\Gamma_{b}$, the black SG of $\Gamma$. Then $\Gamma_{r}$, the red SG of $\Gamma$, equals $\Delta^{*}$. Any color-reversing symmetry $\gamma$ of $\Gamma$ maps the vertices of $\Gamma_{b}$ onto the vertices of $\Gamma_{r}$ and corresponds to an isomorphism between $\Delta$ and $\Delta^{*}$. Conversely assume that there is 
an isomorphism from $\Delta$ to $\Delta^{*}$. That isomorphism extends to an isomorphism of $\Gamma$, as the TFSG constructed on $\Gamma_{b}$, onto $\Gamma$, as the TFSG constructed on $\Gamma_{r}$, and hence to a color-reversing symmetry of $\Gamma$.

From now on we will restrict our attention to special spherical grids (SSGs) defined as follows: a properly colored TFSG, $\Gamma=(V, E, F)$, that admits a color-reversing symmetry. An example of an SSG is pictured on the right in Figure 3. In fact this is the simplest example possible: here both $\Gamma_{b}$ and $\Gamma_{r}$ are copies of the tetrahedral graph. Visualizing $\Gamma$ as drawn on the sphere, the antipodal map is one of the several color-reversing symmetries of $\Gamma$.

\section{Local Coordinates on an SSG}

In order to investigate the special spherical grids, we alter $\Lambda$, the rectangular tessellation of the plane, as follows: we identify the vertices of $\Lambda$ with the set of points in $\mathcal{R}^{2}$ that have integer coordinates. Integer points with both coordinates even are colored black; points with both coordinates odd are colored red and the remaining points with integer coordinates are colored white. Edges on horizontal lines with even y-intercept and edges on vertical lines with even x-intercept are colored black; all other edges are colored red. In constructing our templates in $\Lambda$, we will use the coordinate system just described. However, once the template is extracted and the identifications made, these $\Lambda$-coordinates no longer make sense. Hence we introduce a system of $S S G$-coordinates relating nearby vertices.

We use the term segment for the straight line segment joining two vertices in $\Lambda$. The $\Lambda$-coordinates of a directed segment are simply the coordinates of the vector from the first vertex to the second vertex. The SSG-coordinates of the segment are obtained as follows: choose a new coordinate system for $\Lambda$ with one endpoint of the segment at the origin and the other endpoint on the positive $x$-axis or in the first quadrant. The $\Lambda$-coordinates of the second endpoint are then the SSG-coordinates of the segment: $(x, y)$ or simply $(x)$ for $(x, 0)$, if the second endpoint is on the $x$-axis. See Figure 4 . The advantages of these SSG-coordinates are that they are independent of the orientation of the segment and that in some cases segments and their SSG coordinates can be defined on an SSG.

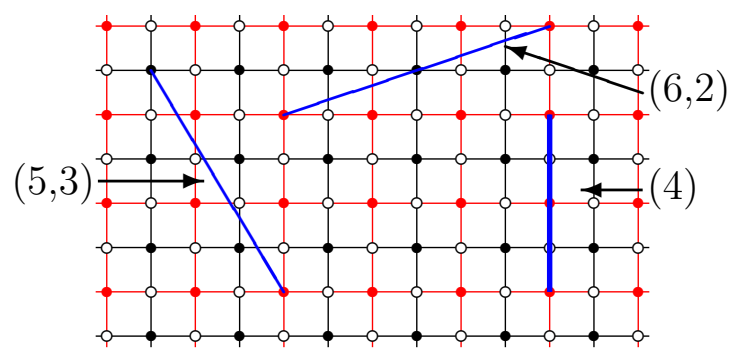

Figure 4: The assignment of coordinates to segments in $\Lambda$. 
We say that two vertices $v$ and $u$ are adjacent on an SSG $\Gamma$ if there are no degree-3 vertices (other than possibly $v$ or $u$ ) on any shortest path from $u$ to $w$. Suppose that vertices $v$ and $u$ are adjacent on the SSG $\Gamma$ and let $v=v_{0}, v_{1}, \ldots, v_{n}, v_{n+1}=u$ be a shortest path joining $v$ and $u$. Since, for $i=1, \ldots, n, v_{i}$ has degree 4 , we may identify the path $v_{i-1}, v_{i}, v_{i+1}$ as a right turn, left turn or a straight-on path. There are several observations that can be easily verified:

i. Since this is a shortest path in $\Gamma$, left and right turns must alternate.

ii. Starting with adjacent vertices $v_{0}^{\prime}$ and $v_{1}^{\prime}$ in $\Lambda$, we may trace a path $v_{0}^{\prime}, v_{1}^{\prime}, \ldots, v_{n}^{\prime}, v_{n+1}$ with the corresponding turns.

iii. The vertices $v^{\prime}=v_{0}^{\prime}$ and $u^{\prime}=v_{n+1}$ in $\Lambda$ then determine a rectangle that contains all shortest paths from $v^{\prime}$ to $u^{\prime}$.

iv. Working out from our initial path in $\Gamma$ we may construct a copy of this rectangle in $\Gamma$ without encountering vertices of degree 3 .

v. Hence we may think of $v$ and $u$ as being joined by a segment in $\Gamma$ with the SSG coordinates of the segment joining $v^{\prime}$ and $u^{\prime}$ in $\Lambda$.

Next we note that if $v$ and $u$ and $\bar{v}$ and $\bar{u}$ are two pairs of points in $\Lambda$, then there is a symmetry of $\Lambda$ mapping the first pair onto the second pair if and only if the segments joining pairs have the same or reversed SSG-coordinates. Furthermore we note that when $p \neq q$, the rectangle of a segment with coordinates $(p, q)$ and the rectangle of a segment with coordinates $(q, p)$ have different orientation and that any symmetry of $\Lambda$ mapping one onto the other must be orientation reversing. It follows that an automorphism of $\Gamma$ will map pairs of adjacent vertices onto pairs of adjacent vertices. In that case, the segments of the original pair and the image pair have the same coordinates or reversed coordinates and in the former case the automorphism would be orientation preserving while in the latter case orientation reversing. For later reference, we we formalize this discussion as a lemma.

Lemma 4. Let $\Gamma$ be a $S S G$, let $u$ and $w$ be a pair of adjacent vertices joined a segment with $S S G$-coordinates $(p, q)$. Let $\phi$ be a symmetry of $\Gamma$. Then $\phi(u)$ and $\phi(w)$ are adjacent and the segment joining them has $S S G$-coordinates $(p, q)$ when $\phi$ is orientation-preserving and $(q, p)$ when $\phi$ is orientation-reversing.

Since we will be constructing our templates in a copy of $\Lambda$ with a fixed coordinate system, we will frequently need to convert between $\Lambda$-coordinates and SSG-coordinates; the following easily proved lemma gives the conversion formulas.

Lemma 5. Let $u$ and $v$ be two vertices of $\Lambda$ and let $(x, y)$ be the $\Lambda$-coordinates of the vector from $v$ to $u$. Then the $S S G$-coordinates $(p, q)$ of the segment joining $v$ and $u$ are computed as follows:

i. $(p, q)=(x, y)$ when $x>0$ and $y>0 ;$ 


$$
\begin{aligned}
& \text { ii. }(p, q)=(y,-x) \text { when } x<0 \text { and } y>0 \text {; } \\
& \text { iii. }(p, q)=(-y, x) \text { when } x>0 \text { and } y<0 \text {; } \\
& \text { iv. }(p, q)=(-x,-y) \text { when } x<0 \text { and } y<0 \text {. }
\end{aligned}
$$

Furthermore, if $x y=0$, the $S S G$-coordinates $(p)$ is the absolute value of the non-zero $\Lambda$-coordinate.

In $\Lambda$ there are two natural ways to measure the distance between vertices or lengths for segments: the graph theory length of a segment with SSG-coordinates $(p, q)$ is simply $p+q$ while the geometric length of a segment with $\operatorname{SSG-coordinates}(p, q)$ is $\sqrt{p^{2}+q^{2}}$. Given a vertex of an SSG, we will frequently consider the set of degree-3 vertices nearest to it. By that we will mean those degree-3 vertices that are adjacent to the given vertex by the graph-theoretical shortest segments among the geometrically shortest segments. For example, if the vertices adjacent to the vertex $v$ have segments with SSG-coordinates $(10,15),(1,18)$ and $(6,17)$ all are a geometric distance of $\sqrt{325}$ from $v$, but the vertex with SSG-coordinates $(1,18)$ is nearest to $v$ with a graph theory distance of 19 ; on the other hand, if the vertices adjacent to $v$ by segments with SSG-coordinates $(1,12),(5,8)$ and $(6,7)$ all are a graph theory distance of 13 from $v$, but the vertex with SSG-coordinates $(6,7)$ is nearest to $v$ with a geometric distance of $\sqrt{85}$. Suppose that in an SSG $u$ and $\bar{u}$ are both among the degree- 3 vertices nearest to $v$ and assume that the segments have coordinates $(p, q)$ and $(\bar{p}, \bar{q})$ respectively. Then these coordinates satisfy the system

$p+q=\bar{p}+\bar{q}$ and $\sqrt{p^{2}+q^{2}}=\sqrt{\bar{p}^{2}+\bar{q}^{2}}$ and hence either $\bar{p}=p$ and $\bar{q}=q$ or $\bar{p}=q$ and $\bar{q}=p$. Again we formulate this observation as a lemma for later reference.

Lemma 6. Let $\Gamma$ be an $S S G$ and let $v$ be a vertex. Then all segments to the nearest degree-3 neighbors of $v$ have $S S G$-coordinates $(p, q)$ or $(q, p)$ for a fixed $p$ and $q$.

\section{Regions of an SSG}

By definition all of the faces of an SSG are squares. Hence if the SSG is given by a template in $\Lambda$, the number of faces of the SSG is simply the total area of its template. Based on this observation we will be able to compute the number of vertices in a self-dual spherical grid in terms of the area of the template of the corresponding SSG.

Lemma 7. Let $\Gamma=\left(V_{b} \cup V_{r} \cup V_{w}, E, F\right)$ be an $S S G$ and let $A$ denote the number of faces of $\Gamma$ or the area of its template. Then $\left|V_{b}\right|=\left|V_{r}\right|=\frac{1}{4} A+1$.

Proof. Counting edges from the red-black side of the bipartition $\left[V_{b} \cup V_{r}, V_{w}\right]$ gives $|E|=$ $\left(4\left|V_{b}\right|-4\right)+\left(4\left|V_{r}\right|-4\right)=4\left(\left|V_{b}\right|+\left|V_{r}\right|-2\right)$. On the other hand each face is bounded by 4 edges; so, $2|E|=4|F|$. Combining these two equations yields $2\left(\left|V_{b}\right|+\left|V_{r}\right|-2\right)=|F|$. Finally since $\Gamma_{b}$ is self-dual $\left|V_{b}\right|=\left|V_{r}\right|$ and we have $4\left|V_{b}\right|-4=|F|=A$. 
One basic region that will occur regularly in our investigation is that of a parallelogram. Specifically, most of our SSG templates will be partitioned into parallelograms with degree-3 vertices at the vertices of the parallelograms. Each parallelogram $\mathcal{P}$ can be embedded in $\Lambda$ with one vertex $v_{0}$ at the origin. Let $v_{1}=\left(x_{1}, y_{1}\right)$ and $v_{3}=\left(x_{3}, y_{3}\right)$ be the vertices of $\mathcal{P}$ adjacent to $v_{0}$. Then from basic vector geometry, we have that $v_{2}$, the vertex opposite $v_{0}$, has $\Lambda$-coordinates $\left(x_{1}+x_{3}, y_{1}+y_{3}\right)$. The matrix $\left[\begin{array}{ll}x_{1} & x_{3} \\ y_{1} & y_{3}\end{array}\right]$ gives the transformation of the plane that maps the unit square onto the parallelogram. Hence the absolute value of its determinate, $\left|x_{1} y_{3}-x_{3} y_{1}\right|$, is the area of the parallelogram. If $x_{1} y_{3}-x_{3} y_{1}$ is positive then the transformation preserves orientation and $v_{1}, v_{3}$ are in counterclockwise order about $v_{0}$; if $x_{1} y_{3}-x_{3} y_{1}$ is negative then $v_{1}, v_{3}$ are in clockwise order.

Our basic method for constructing an SSG from a template will be to form a "cylinder" sandwiched between two "caps." Let $v_{0}, v_{1}, v_{2}$ and $v_{3}$ be four vertices in $\Lambda$ and let $\mathcal{H}$ denote their convex hull. If $\mathcal{H}$ is a quadrilateral, we assume that $v_{0}, v_{1}, v_{2}$ and $v_{3}$ occur in counterclockwise order around the boundary of $\mathcal{H}$. If $\mathcal{H}$ is a triangle, we assume that $v_{1}, v_{2}$ and $v_{3}$ occur in counterclockwise order with $v_{0}$ interior to $\mathcal{H}$. If $\mathcal{H}$ is a straight line segment, we assume that $v_{0}, v_{1}, v_{2}$ and $v_{3}$ occur in counterclockwise order with $v_{0}$ and $v_{2}$ as the endpoints of the segment.

Assume first that $\mathcal{H}$ is a quadrilateral or a straight line segment. Now using an elementary geometric argument one can construct disjoint $90^{\circ}$ wedges at the vertices so that the left bounding ray at $v_{0}$ is parallel to the right bounding ray at $v_{1}$, the left bounding ray at $v_{1}$ is parallel to the right bounding ray at $v_{2}$ and so on. In the case that $\mathcal{H}$ is a triangle, we note that the angles $\angle v_{1} v_{0} v_{2}, \angle v_{2} v_{0} v_{3}$ and $\angle v_{3} v_{0} v_{1}$ sum to 360 degrees. Hence we may assume that the angle $\angle v_{1} v_{0} v_{3}$ is greater than $90^{\circ}$ and we may choose a $90^{\circ}$ wedge with vertex $v_{0}$ that separates $v_{1}$ and $v_{3}$ and then proceed to construct wedges at the other vertices with consecutive sides parallel. Since the counterclockwise rotation by $90^{\circ}$ about any vertex of $\Lambda$ is a symmetry of $\Lambda$, the vertices, edges and faces match up when a wedge is removed and its bounding rays identified. Carrying out this deletion and identification at each vertex results in an infinite cylinder with a "cap" containing $\mathcal{H}$ at the end. A typical configuration is pictured in Figure 5. Segments of constant slope joining a point on the cylinder to itself are all parallel and have the same length. One of these segments connects one of the $v_{0}, v_{1}, v_{2}$ and $v_{3}$ to itself and is called the rim of the cylinder; the region it bounds is called the cap. This rim is also called rim of the cap.

Consider the cap $\mathcal{C}$ and its $\operatorname{rim} \mathcal{R}$. To continue this construction and eventually produce a template, we must take into account that an SSG has four more degree-3 vertices. One of them will be among the "closest" to $\mathcal{R}$. The segment parallel to $\mathcal{R}$ through that vertex is then the rim of the "bottom" cap and the region between the two rims is the actual cylinder of this template. This bottom cap, like the top cap will be a finite region, very often isomorphic to the top cap. Finally, if the cylinder is slit open along a segment connecting a degree- 3 vertex on one rim to a degree- 3 vertex on the other rim and unrolled we see that it is a parallelogram.

To compute the SSG-coordinates of the rim, place $v_{0}$ at the origin. Assume that the 

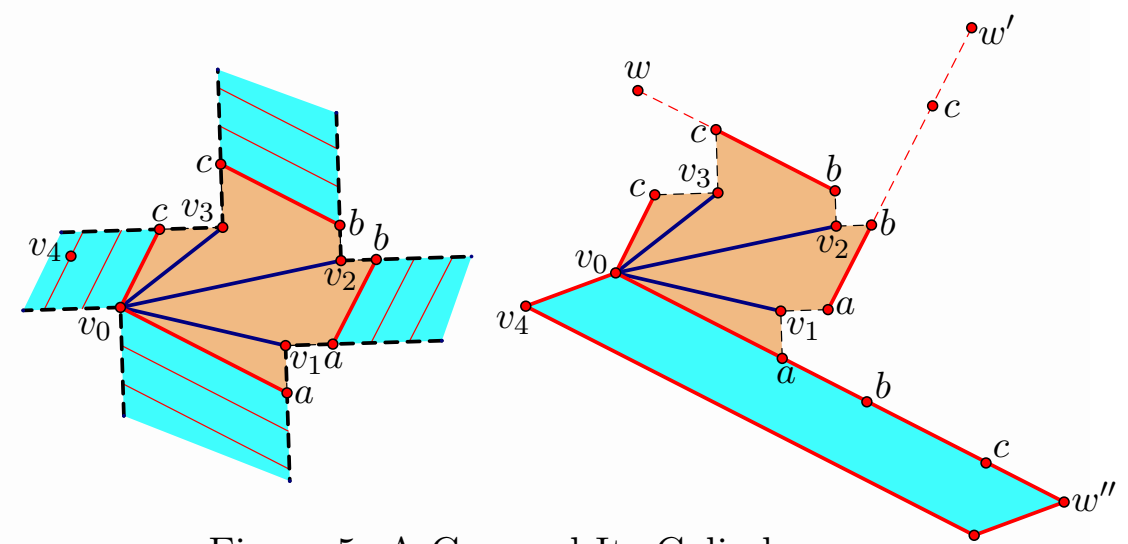

Figure 5: A Cap and Its Cylinder.

$\Lambda$-coordinates of $v_{i}$ are $\left(x_{i}, y_{i}\right)$ and that $v_{1}$ is in the first quadrant. Now let $w$ denote the image of the origin under the $90^{\circ}$ clockwise rotation about $v_{3}$, let $w^{\prime}$ denote the image of $w$ under the $90^{\circ}$ clockwise rotation about $v_{2}$ and let $w^{\prime \prime}$ denote the image of $w^{\prime}$ under the $90^{\circ}$ clockwise rotation about $v_{1}$. One easily checks that the $\Lambda$-coordinates of $w^{\prime \prime}$ are

$$
\left(x_{1}-y_{1}+x_{2}+y_{2}-x_{3}+y_{3}, x_{1}+y_{1}-x_{2}+y_{2}-x_{3}-y_{3}\right) \text {. }
$$

When wedges are removed and the cylinder reconstituted the segment $v_{0}-w^{\prime \prime}$ becomes the rim of the cap. The $\Lambda$-coordinates of $w^{\prime \prime}$ converted to the SSG-coordinate format are called the rim coordinates of the cylinder or cap.

In general, computing the area of the cap is rather complicated. However, there are only two cases for which we will actually use this cap-cylinder-cap decomposition, and we now compute the cap area for these two cases. The two cases are: caps about the vertices of parallelograms and caps with rims on $45^{\circ}$ lines; representatives of these two types are pictured in Figure 6. In these cases, one can easily choose the wedges so that their sides are perpendicular to the rim.

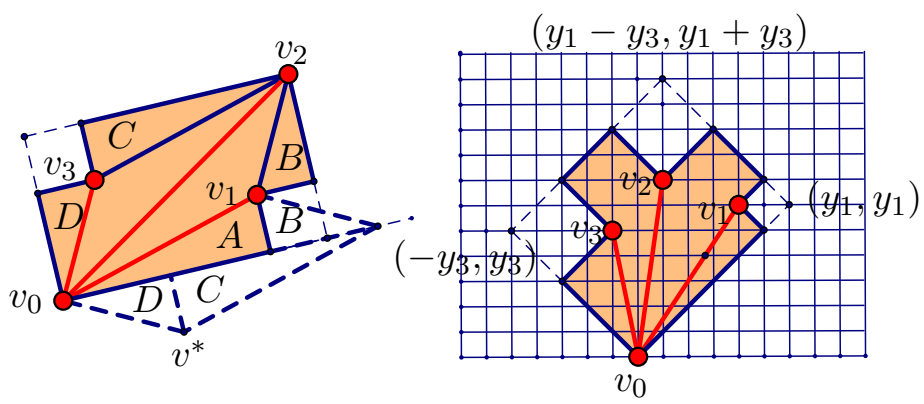

Figure 6: Special Cases.

First consider the parallelogram (on the left in the figure); here the wedges have been chosen so that their sides are perpendicular to the rim. Substituting $x_{1}+x_{3}$ for $x_{2}$ and $y_{1}+y_{3}$ for $y_{2}$ in the rim formula gives $\left(2 x_{1}+2 y_{3}, 2 y_{1}-2 x_{3}\right)$ for the rim coordinates. To compute the cap area, we start with the parallelogram itself; it has area $x_{1} y_{3}-y_{1} x_{3}$. Let $v^{*}$ be the image of $v_{3}$ under the $90^{\circ}$ clockwise rotation about $v_{0}$. Observe that the four triangular regions of the cap outside of the parallelogram (labeled $A, B, C$ and $D$ ) may 
be assembled into the parallelogram given by vectors $v^{*}$ and $v_{1}$. The $\Lambda$-coordinates of $v^{*}$ are $\left(y_{3},-x_{3}\right)$; hence the area of this parallelogram is $y_{3} y_{1}+x_{1} x_{3}$ and the area of the cap is $x_{1} y_{3}-y_{1} x_{3}+y_{3} y_{1}+x_{1} x_{3}$.

Turning to the $45^{\circ}$ case and setting the rim coordinates equal to one another, we have $\left(x_{1}-y_{1}+x_{2}+y_{2}-x_{3}+y_{3}\right)=\left(x_{1}+y_{1}-x_{2}+y_{2}-x_{3}-y_{3}\right)$. This gives $x_{2}=$ $y_{1}-y_{3}$. Substituting $y_{1}-y_{3}$ for $x_{2}$ in the rim coordinates gives the rim coordinates $\left(x_{1}+y_{2}-x_{3}, x_{1}+y_{2}-x_{3}\right)$ in this case. Turning to area, we easily see that the corners of the rectangle have $\Lambda$-coordinates $\left(y_{1}, y_{1}\right),\left(y_{1}-y_{3}, y_{1}+y_{3}\right)$ and $\left(-y_{3}, y_{3}\right)$ (in counterclockwise order). Hence the entire rectangle has area $2 y_{1} y_{3}$ and we need only subtract off the areas of the three corner squares. The area of a square with $45^{\circ}$ angled sides is simply the square of its height or width divided by 2 . Hence the area of the cap is $2 y_{1} y_{3}-$ $\frac{1}{2}\left(\left(y_{1}-x_{1}\right)^{2}+\left(y_{1}+y_{3}-y_{2}\right)^{2}+\left(y_{3}+x_{3}\right)^{2}\right)$. For easy reference, we summarize all of these geometric results in the next lemma.

Lemma 8. Let $\mathcal{P}$ the cap of a set of four degree-3 vertices in $\Lambda$. Assume that the degree-3 vertices are labeled $v_{0}, v_{1}, v_{2}$ and $v_{3}$ and are arranged in counterclockwise order with $v_{0}$ on the rim. Assume that the coordinate system for $\Lambda$ is chosen with $v_{0}$ at the origin and $v_{1}$ in the first quadrant. Finally, let $v_{4}$ denote the point on the bottom rim nearest to $v_{0}$ and let $v_{i}$ have $\Lambda$-coordinates $\left(x_{i}, y_{i}\right)$ for $i=1, \ldots, 4$. Then:

i. The rim coordinates are

$\left(x_{1}-y_{1}+x_{2}+y_{2}-x_{3}+y_{3}, x_{1}+y_{1}-x_{2}+y_{2}-x_{3}-y_{3}\right)$

(a) If this is a parallelogram cap, $x_{2}=x_{1}+x_{3}, y_{2}=y_{1}+y_{3}$ and the rim coordinates are $\left(2 x_{1}+2 y_{3}, 2 y_{1}-2 x_{3}\right)$.

(b) If this is a $45^{\circ}$ cap, $x_{2}=y_{1}-y_{3}$ and the rim coordinates are $\left(x_{1}+y_{2}-x_{3}, x_{1}+\right.$ $\left.y_{2}-x_{3}\right)$

ii. If $v^{\prime}=\left(x^{\prime}, y^{\prime}\right)$ and $v^{\prime \prime}=\left(x^{\prime \prime}, y^{\prime \prime}\right)$ are vectors, $x^{\prime} y^{\prime \prime}-x^{\prime \prime} y^{\prime}>0$ when $v^{\prime}$ and $v^{\prime \prime}$ are in counterclockwise order around the origin and $x^{\prime} y^{\prime \prime}-x^{\prime \prime} y^{\prime}<0$ when $v^{\prime}$ and $v^{\prime \prime}$ are in clockwise order. In either case $\left|x^{\prime} y^{\prime \prime}-x^{\prime \prime} y^{\prime}\right|$ is the area of the parallelogram with vertices $v_{0}, v^{\prime}, v^{\prime \prime}$ and $v^{\prime}+v^{\prime \prime}$. Thus the area of the cylinder is $\mid x_{4}\left(x_{1}-y_{1}+x_{2}+\right.$ $\left.y_{2}-x_{3}+y_{3}\right)-y_{4}\left(x_{1}+y_{1}-x_{2}+y_{2}-x_{3}-y_{3}\right) \mid$

(a) If this is a parallelogram cap, the area of the cap is $x_{1} y_{3}-x_{3} y_{1}+y_{1} y_{3}+x_{1} x_{3}$ and the area of the cylinder is $2\left|x_{4}\left(y_{1}-x_{3}\right)-y_{4}\left(x_{1}+y_{3}\right)\right|$.

(b) If this is a $45^{\circ}$ cap, the area of the cap is

$2 y_{1} y_{3}-\frac{1}{2}\left(\left(y_{1}-x_{1}\right)^{2}+\left(y_{3}+x_{3}\right)^{2}+\left(y_{1}+y_{3}-y_{2}\right)^{2}\right)$ and the area of the cylinder is $\left|\left(x_{4}-y_{4}\right)\left(x_{1}+y_{2}-x_{3}\right)\right|$. 


\section{The Symmetries and Symmetry Groups of SSGs}

The main result of this section is a complete listing of the symmetries and symmetry groups of the SSGs. A similar study was carried out for general self-dual plane graphs by B. Servatius and H. Servatius [7]. Any plane graph may be embedded on the sphere so that the symmetries of the graph are isometries of the sphere and its symmetry group is a discrete group of isometries on the sphere (See Lemma 1 in [7]). In our investigation of the symmetries and symmetry groups of SSGs, we will use terminology of the isometries of the sphere as in Coxeter and Moser [3]. It is also the case that each symmetry of an SSG must permute the eight degree-3 vertices and therefore may be identified with a subgroup of the permutation group of these vertices. We will also use the terminology of this permutation group. Specifically we will talk about the stabilizers of the degree-3 vertices and the orbits of a symmetry or of a symmetry group meaning the orbits of the permutation or permutation group of the degree-3 vertices. We will frequently use the result that the product of the size of the orbit containing a degree- 3 vertex and the size of its stabilizer equals the order of the symmetry group.

Let $\Gamma=(V, E, F)$ be an SSG. Any symmetry $\gamma$ of $\Gamma$ must respect the bipartition $\left(V_{b} \cup\right.$ $\left.V_{r}, V_{w}\right)$ of its vertex set. Since all of the degree-3 vertices are in $V_{b} \cup V_{r}, \gamma\left(V_{w}\right)=V_{w}$. Any symmetry $\gamma$ must also respect the bipartition of the auxiliary graph and either preserve the subgraphs $\left(V_{b} \cup V_{w}, E_{b}\right)$ and $\left(V_{r} \cup V_{w}, E_{r}\right)\left[\gamma\left(V_{b}\right)=V_{b}\right.$ and $\gamma\left(V_{r}\right)=V_{r}$, color-preserving $]$ or interchange them $\left[\gamma\left(V_{b}\right)=V_{r}\right.$ and $\gamma\left(V_{r}\right)=V_{b}$, color-reversing]. The definition of an SSG requires that $\Gamma$ admit at least one color-reversing symmetry. The symmetries of $\Gamma$ may also be classified as direct (orientation preserving) or opposite (orientation reversing) depending on whether their extension to an isometry of the sphere is direct or opposite. By Lemma 4, the direct symmetries map segments with SSG-coordinates $(p, q)$ onto segments with SSG-coordinates $(p, q)$ while opposite symmetries map segments with SSG-coordinates $(p, q)$ onto segments with SSG-coordinates $(q, p)$.

Lemma 9. Let $\Gamma=\left(V_{b} \cup V_{r} \cup V_{w}, E, F\right)$ be an $S S G$. Let $T_{b}$ and $T_{r}$ denote the degree-3 black and red vertices, respectively. Then any symmetry of $\Gamma$ must be one of the following:

- The direct, color-preserving symmetries:

i. rotations of order 4 about antipodal degree-4 vertices from $V_{b} \cup V_{r}$;

ii. rotations of order 3 about antipodal degree-3 vertices

$c_{b} \in T_{b}$ and $c_{r} \in T_{r}$

iii. rotations of order 2 about antipodal vertices.

- The direct, color-reversing symmetries:

iv. rotations of order 4 about antipodal vertices from $V_{w}$;

$v$. rotations of order 2 about the centers of antipodal faces.

- The opposite, color-preserving symmetries: 
vi. A reflection through a color-preserving equator*;

vii. A rotatory-reflection of order 4 with centers of the same color;

viii. The antipodal map $\alpha$.

- The opposite, color-reversing symmetries:

ix. A reflection with a color-reversing equator*;

$x$. A rotatory-reflection of order 8 with antipodal degree-3 centers $c_{b} \in T_{b}$ and $c_{r} \in T_{r}$ and a color-reversing

equator*;

xi. A rotatory-reflection of order 6 with antipodal degree-3 centers $c_{b} \in T_{b}$ and $c_{r} \in T_{r}$ and a color-reversing equator*;

xii. A rotatory-reflection of order 4 with centers from $V_{w}$ or one red and one black center and a color-reversing equator*;

xiii. The antipodal map $\alpha$.

${ }^{*}$ Color-preserving and color-reversing equators are listed in Figure $\%$.

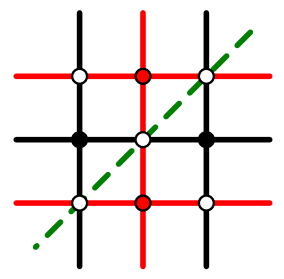

color-reversing

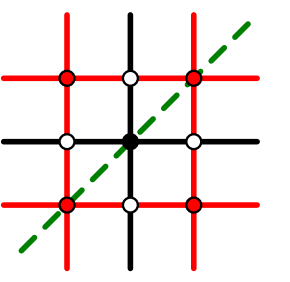

$\longleftarrow$ color-preserving $\longrightarrow$

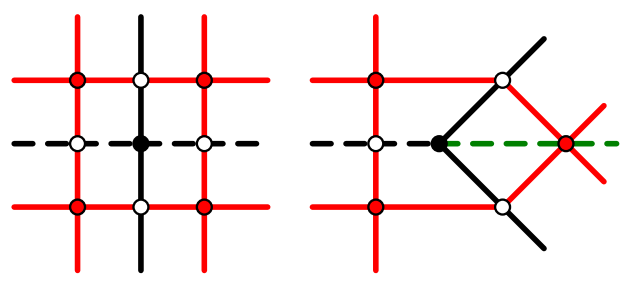

Figure 7: Possible equators for a reflection.

Proof. Since $\Gamma$ is a plane graph any symmetry of $\Gamma$ corresponds to an isometry of the sphere. Hence, the direct symmetries of $\Gamma$ must be rotations and have two antipodal fixed points. We call these fixed points the centers of the rotation. Since these rotations are also graph symmetries, the only choices for centers are: vertices, centers of faces and centers of edges. It follows that the only possible orders for a rotation are 2,3 or 4 (for vertices), 2 or 4 (for faces) and 2 (for edges). Since a rotation of order 2 about the center of an edge interchanges a white vertex with a red or black vertex, it cannot extend to a symmetry of $\Gamma$. Since a rotation of order 4 about the center of a face maps the white vertices bounding the face onto the black and red vertices bounding that face, it also cannot extend to a symmetry of $\Gamma$. Hence the only possibilities are rotations of order 2 , 3 or 4 centered at vertices or half-turns (order 2) centered at faces. 
A rotation of order 4 about a red or black vertex is color-preserving while a rotation of order 4 about a white vertex is color-reversing. Hence, rotations of order 4 have both centers in $V_{w}$ [item (iv) above] or both centers in $V_{b} \cup V_{r}$ [item (i)]. The two centers of a rotation of order 3 can only be vertices of degree-3. The remaining six vertices of degree-3 must fall into two orbits of length 3 each. Since there are 4 red and 4 black vertices of degree-3, there must one red and one black center and one red and one black orbit of length 3 [item (ii)]. A rotation of order 2 about the center of a face interchanges the white vertices bounding the face and interchanges the black and red vertices bounding that face; so it could only extend to a color-reversing symmetry of $\Gamma$ [item (v)]. A rotations of order 2 about a vertex of any color is color-preserving [item (iii)].

The only possible opposite symmetries are reflections and rotatory-reflections. Reflections of the sphere have a plane of reflection which intersects the sphere in a great circle or equator that is fixed. If $\rho$ is a reflection of $\Gamma$, the equator of its extension to the sphere can intersect a face of $\Gamma$ only in an edge (in which case it interchanges the faces containing that edge) or a diagonal of the face (in which case it maps that face onto itself). If the equator of a reflection coincides with the diagonal of a face $f$ joining two white vertices $v$ and $w$, then that equator must include the white-white diagonals of the faces containing $v$ and $w$ but not adjacent to $f$. It follows that, if an equator contains one white-white diagonal it must be an entire circuit of white-white diagonals. In this case, the reflection is color-reversing [item (ix)]. We will refer to such a circuit of white-white diagonals as a color-reversing equator. See the left-hand diagram in Figure 7.

If the equator of a reflection coincides with the diagonal of a face $f$ joining red and black vertices $v$ and $w$ of degree 4 , then that equator must include the red-black diagonals of the faces containing $v$ and $w$ but not adjacent to $f$. See Figure 7. Now assume that the equator of a reflection coincides with the black (red) edge joining the white vertex $v$ and the black (red) vertex $w$. Then that equator must include the other black (red) edge containing $v$. When $w$ has degree 4 , the equator must include the opposite black (red) edge containing $w$; when $w$ has degree-3, the equator must include the red-black diagonal of the face containing the other two edges at $w$. In all of these cases, the reflection is color-preserving [item (vi)]. We conclude that a color-preserving equator is a circle of edges and red-black diagonals as pictured in the right three diagrams of Figure 7.

Each rotatory-reflection $\gamma$ in the symmetry group of $\Gamma$ is the composition of a rotation $\sigma$ and a reflection $\rho$ where the plane of its equator is perpendicular to the axis of $\sigma$. If both $\sigma$ and $\rho$ are themselves symmetries of $\Gamma$, the center and equator of $\gamma$ are well defined. However, $\gamma=\sigma \rho$ may be a symmetry of $\Gamma$ even when $\sigma$ and $\rho$ are not. Since the axis of $\sigma$ is perpendicular to the plane of $\rho, \sigma$ and $\rho$ commute. Thus, $\gamma=\sigma \rho=\rho \sigma$ and $\gamma^{2}=\sigma^{2}$. Hence if $\sigma$ has order greater than 2, we may still recover the center and equator for $\gamma$ : the centers of $\gamma$ are the centers of the rotation $\sigma^{2}$ and the equator of $\gamma$ is the perpendicular plane bisecting the axis of $\sigma$. In the case that $\sigma$ is a half-turn, $\sigma^{2}$ and hence $\gamma^{2}$ is the identity map and there is no uniquely defined axis nor uniquely defined equator. In this case, $\gamma$ is the unique antipodal symmetry and is denoted by $\alpha$. For each vertex, edge or face $X, \alpha(X)$ is called the antipodal vertex, edge or face of $X$; the antipodal map $\alpha$ may be either color-preserving [item (viii)] or color-reversing [item (xiii)]. 
Suppose that $\gamma$ is a rotatory-reflection of order $n>2$. Then $\gamma^{2}$ is a rotation of order $\frac{n}{2}$. Hence $n=4,6$ or 8 . If $n$ is 8 then the vertices of degree- 3 must form an orbit of length 8 . Since there are 4 red and 4 black vertices of degree-3, $\gamma$ must be color-reversing. However, $\gamma^{2}$ is a color-preserving rotation of order 4. Hence, the centers of $\gamma$ must be in $V_{b} \cup V_{r}$, and since $\gamma$ interchanges the centers, one is red and the other is black [item (x)].

Using the same reasoning, we conclude that a rotatory-reflection $\gamma$ of order 6 is colorreversing and has an orbit consisting of three red and three black degree- 3 vertices. The remaining two vertices of degree- 3 must be the degree- 3 red and the degree- 3 black centers of the rotation $\gamma^{2}$ [item (xi)].

Finally, the square of a rotatory-reflection of order 4 is a color-preserving half-turn; hence its centers are vertices. Therefore, a color-preserving rotatory-reflection of order 4 must have both centers of the same color [item (vii)] and color-reversing rotatoryreflection of order 4 must have one red center and one black center or both centers white [item (xii)].

Since the composition of two color-preserving symmetries is also color-preserving and the composition of two color-reversing symmetries is a color-preserving symmetry, the color-preserving symmetries form an index 2 subgroup. This subgroup may be identified with the symmetry group of the subgraph $\left(V_{b} \cup V_{w}, E_{b}\right)$ and the underlying self-dual spherical grid $\Gamma_{b}$. So, in Theorem 1 below, we list a pair: the symmetry group of the SSG, $\Gamma$, and the symmetry group of the underlying self-dual spherical grid, $\Gamma_{b}$. This result is similar to Theorem 1 in Servatius and Servatius [7]. The main difference is that here the groups are specifically tied to spherical grids and then to the templates. A few of our groups are consolidated into a single class in [7].

Theorem 1. Following is a complete list of all possible symmetry groups $\mathcal{A}_{\Gamma}$ of an $S S G$ $\Gamma$ along with the induced symmetry group $\mathcal{A}_{\Delta}$ of $\Delta$, the underlying self-dual $S G$.

- Order 2

i. $\mathcal{A}_{\Gamma}=\{$ one color-reversing reflection $\rho, \iota\} ; \mathcal{A}_{\Delta}=\{\iota\}$.

ii. $\mathcal{A}_{\Gamma}=\{$ the color-reversing antipodal map $\alpha, \iota\} ; \mathcal{A}_{\Delta}=\{\iota\}$.

iii. $\mathcal{A}_{\Gamma}=\{$ one color-reversing half-turn $\tau, \iota\} ; \mathcal{A}_{\Delta}=\{\iota\}$.

- Order 4

iv. $\mathcal{A}_{\Gamma}=\left\{\right.$ color-reversing reflections, $\rho_{1}$ and $\rho_{2}$, one half-turn $\tau=\rho_{1} \rho_{2}$, colorpreserving, and $\iota\} . \mathcal{A}_{\Delta}=\{\tau, \iota\}$.

$v$. $\mathcal{A}_{\Gamma}=\{$ reflections $\rho$, color-reversing, $\eta$, color-preserving, the color-reversing half-turn $\tau=\rho \eta$ and $\iota\} . \mathcal{A}_{\Delta}=\{\eta, \iota\}$.

vi. $\mathcal{A}_{\Gamma}=\{$ One color-reversing reflection $\rho$, one color-preserving half-turn $\tau$, the color-reversing antipodal map $\alpha=\rho \tau$ and $\iota\} . \mathcal{A}_{\Delta}=\{\tau, \iota\}$.

vii. $\mathcal{A}_{\Gamma}=\{$ One color-reversing reflection $\rho$, one color-reversing half-turn $\tau$, the color-preserving antipodal map $\alpha=\rho \tau$ and $\iota\} . \mathcal{A}_{\Delta}=\{\alpha, \iota\}$. 
viii. $\mathcal{A}_{\Gamma}=\{$ One color-preserving reflection $\eta$, one color-reversing half-turn $\tau$, the color-reversing antipodal map $\alpha=\eta \tau$ and $\iota\} . \mathcal{A}_{\Delta}=\{\eta, \iota\}$.

ix. $\mathcal{A}_{\Gamma}=\{$ One color-reversing rotatory-reflection $\phi$ of order 4 and its powers $\}$. $\mathcal{A}_{\Delta}=\left\{\phi^{2}, \iota\right\}$.

x. $\mathcal{A}_{\Gamma}=\{$ Three half-turns with pairwise orthogonal axes: two color-reversing, $\tau_{1}, \tau_{2}$ and one color-preserving, $\left.\tau_{3}=\tau_{1} \tau_{2}\right\} . \mathcal{A}_{\Delta}=\left\{\tau_{3}, \iota\right\}$.

- Order 6

xi. $\mathcal{A}_{\Gamma}=\{$ One color-preserving rotation $\sigma$ of order 3 and its powers and three color-reversing half-turns $\left.\tau_{1}, \tau_{2}, \tau_{3}\right\} . \mathcal{A}_{\Delta}=\left\{\sigma, \sigma^{2}, \iota\right\}$.

- Order 8

xii. $\mathcal{A}_{\Gamma}=\left\{\right.$ One color-preserving rotation $\sigma$ of order 4 and its powers and $\tau_{1}, \tau_{2}, \tau_{3}$, $\tau_{4}$, four color-reversing half-turns $\} . \mathcal{A}_{\Delta}=\left\{\sigma, \sigma^{2} \sigma^{3}, \iota\right\}$.

xiii. $\mathcal{A}_{\Gamma}=\{$ One color-reversing rotation $\sigma$ of order 4 and its powers, two colorreversing half-turns $\tau_{1}$ and $\tau_{3}$, two color-preserving half-turns $\tau_{2}$ and $\left.\tau_{4}\right\} . \mathcal{A}_{\Delta}$ $=\left\{\sigma^{2}, \tau_{2}, \tau_{4}, \iota\right\}$.

xiv. $\mathcal{A}_{\Gamma}=\left\{3\right.$ color-reversing reflections $\rho_{1}, \rho_{2}$ and $\rho_{3}$, the (color-reversing) antipodal map $\alpha=\rho_{1} \rho_{2} \rho_{3}$, three color-preserving half-turns $\tau_{1}=\rho_{1} \rho_{2}, \tau_{2}=\rho_{1} \rho_{3}$, $\tau_{3}=\rho_{2} \rho_{3}$, and the identity. $\} . \mathcal{A}_{\Delta}=\left\{\tau_{1}, \tau_{2}, \tau_{3}, \iota\right\}$.

xv. $\mathcal{A}_{\Gamma}=\left\{2\right.$ color-reversing reflections, $\rho_{1}, \rho_{2}$, and one color-preserving reflection, $\eta$, the (color-preserving) antipodal map $\alpha=\rho_{1} \rho_{2} \eta$, two color-reversing halfturns $\tau_{1}=\rho_{1} \eta, \tau_{2}=\rho_{2} \eta$, one color-preserving half-turn $\tau=\rho_{1} \rho_{2}$, and the identity. $\} . \mathcal{A}_{\Delta}=\{\eta, \alpha, \tau, \iota\}$.

xvi. $\mathcal{A}_{\Gamma}=\left\{2\right.$ color-preserving reflections $\rho_{1}$ and $\rho_{2}$, one color-reversing reflection, $\rho_{3}$, the color-reversing antipodal map $\alpha=\rho_{1} \rho_{2} \rho_{3}$, two color-reversing halfturns $\tau_{1}=\rho_{2} \rho_{3}, \tau_{2}=\rho_{1} \rho_{3}$, one color-preserving half-turn $\tau_{3}=\rho_{2} \rho_{3}$, and the identity. $\} . \mathcal{A}_{\Delta}=\left\{\tau_{3}, \rho_{1}, \rho_{2}, \iota\right\}$.

xvii. $\mathcal{A}_{\Gamma}=\{$ a color-preserving rotatory-reflection $\sigma$ of order 4 and its powers, two color-reversing half-turns $\tau_{1}$ and $\tau_{2}$ and two color-reversing reflections $\rho_{1}$ and $\rho_{2}$ where $\tau_{1} \tau_{2}=\rho_{1} \rho_{2}=\sigma^{2} . \mathcal{A}_{\Delta}=\left\{\iota, \sigma, \sigma^{2}, \sigma^{3}\right\}$.

xviii. $\mathcal{A}_{\Gamma}=\{$ a color-reversing rotatary-reflection $\sigma$ of order 4 and its powers, two color-preserving half-turns $\tau_{1}$ and $\tau_{2}$ and two color-reversing reflections $\rho_{1}$ and $\rho_{2}$ where $\tau_{1} \tau_{2}=\rho_{1} \rho_{2}=\sigma^{2} . \mathcal{A}_{\Delta}=\left\{\iota, \sigma^{2}, \tau_{1}, \tau_{2}\right\}$.

xix. $\mathcal{A}_{\Gamma}=\{$ a color-reversing rotatary-reflection $\sigma$ of order 4 and its powers, two color-reversing half-turns $\tau_{1}$ and $\tau_{2}$ and two color-preserving reflections $\rho_{1}$ and $\rho_{2}$ where $\tau_{1} \tau_{2}=\rho_{1} \rho_{2}=\sigma^{2} . \mathcal{A}_{\Delta}=\left\{\iota, \sigma^{2}, \rho_{1}, \rho_{2}\right\}$.

- Order 12 
$x x . \mathcal{A}_{\Gamma}=\{$ One color-preserving rotation $\sigma$ of order 3 and its powers, three colorreversing half-turns, $\tau_{1}, \tau_{2}, \tau_{3}$, three color-preserving reflections $\rho_{1}, \rho_{2}$ and $\rho_{3}$ through planes containing the axis of $\sigma$, one color-reversing reflection $\rho_{0}$ through the plane perpendicular to the axis of $\sigma$ plus $\sigma \rho_{0}$ and $\left.\sigma^{2} \rho_{0}\right\}$.

$\mathcal{A}_{\Delta}=\left\{\rho_{1}, \rho_{2}, \rho_{3}, \sigma, \sigma^{2}, \iota\right\}$.

xxi. $\mathcal{A}_{\Gamma}=\{$ One color-reversing rotatory-reflection $\phi$ of order 6 and its powers, three color-reversing half-turns, three color-preserving reflections $\rho_{1}, \rho_{2}, \rho_{3}$ through planes containing the axis of $\phi\} . \mathcal{A}_{\Delta}=\left\{\rho_{1}, \rho_{2}, \rho_{3}, \phi^{2}, \phi^{4}, \iota\right\}$.

- Order 16

xxii. $\mathcal{A}_{\Gamma}=\{$ One color-reversing rotatory-reflection $\phi$ of order 8 and its powers, four color-reversing half-turns, four color-preserving reflections $\rho_{1}, \rho_{2}, \rho_{3}, \rho_{4}$ through planes containing the axis of $\phi\} . \mathcal{A}_{\Delta}=\left\{\rho_{1}, \rho_{2}, \rho_{3}, \rho_{4}, \phi^{2}, \phi^{4}, \phi^{6}, \iota\right\}$.

xxiii. $\mathcal{A}_{\Gamma}=\{$ One color-preserving rotation $\sigma$ of order 4 and its powers, four colorreversing half-turns, four color-preserving reflections, $\rho_{1}, \rho_{2}, \rho_{3}, \rho_{4}$, through the planes containing the axis of $\sigma$, one color-reversing reflection $\rho$ through the plane perpendicular to the axis of $\sigma$ plus the rotatory-reflections $\sigma \rho, \sigma^{2} \rho$ and $\left.\sigma^{3} \rho\right\} . \mathcal{A}_{\Delta}=\left\{\rho_{1}, \rho_{2}, \rho_{3}, \rho_{4}, \sigma, \sigma^{2}, \sigma^{3}, \iota\right\}$.

xxiv. $\mathcal{A}_{\Gamma}=\{$ One color-reversing rotation $\sigma$ of order 4 and its powers, two colorreversing half-turns $\tau_{1}$ and $\tau_{3}$, two color-preserving half-turns $\tau_{2}$ and $\tau_{4}$, two color-reversing reflections $\rho_{1}$ and $\rho_{3}$, color-preserving reflections $\rho_{2}$ and $\rho_{4}$ all through planes containing the axis of $\sigma$, one color-preserving reflection $\rho$ with equator perpendicular to the axis of $\sigma$ and its compositions with the powers of $\sigma\} . \mathcal{A}_{\Delta}=\left\{\tau_{2}, \tau_{4}, \rho_{2}, \rho_{4}, \rho, \sigma^{2}, \alpha=\sigma^{2} \rho, \iota\right\}$.

xxv. $\mathcal{A}_{\Gamma}=\{$ One color-reversing rotation $\sigma$ of order 4 and its powers, two colorreversing half-turns $\tau_{1}$ and $\tau_{3}$, two color-preserving half-turns $\tau_{2}$ and $\tau_{4}$, two color-reversing reflections $\rho_{1}$ and $\rho_{3}$, color-preserving reflections $\rho_{2}$ and $\rho_{4}$ all through planes containing the axis of $\sigma$, one color-reversing reflection $\rho$ with equator perpendicular to the axis of $\sigma$ and its compositions with the powers of $\sigma\} . \mathcal{A}_{\Delta}=\left\{\tau_{2}, \tau_{4}, \rho_{2}, \rho_{4}, \sigma \rho, \sigma^{2}, \sigma^{3} \rho, \iota\right\}$.

- Order 24

xxvi. $\mathcal{A}_{\Gamma}=$ the direct symmetry group of the cube and $\mathcal{A}_{\Delta}=$ the direct symmetry group of the tetrahedra.

- Order 48

xxvii. $\mathcal{A}_{\Gamma}=$ the full symmetry group of the cube and $\mathcal{A}_{\Delta}=$ the full symmetry group of the tetrahedra. 
Proof. We will actually prove only half of the theorem here. Namely that this is a complete list of possibilities. The fact that each group does occur will be established in the last section where the smallest example of each type will be constructed.

We start by noting that either all of the symmetries of an SSG are direct or the direct symmetries form an index-2 subgroup. We also note that the composition of two halfturns is a rotation by twice the angle between their axes about an axis perpendicular to the plane containing their axes. Hence, if the SSG admits no rotations of order 3 or 4, its direct subgroup is one of the following:

a. the identity, $\{\iota\}$;

b. one half-turn and the identity, $\{\iota, \tau\}$;

c. three half-turns with pairwise orthogonal axes and the identity, $\left\{\iota, \tau_{1}, \tau_{2}, \tau_{3}\right\}$.

It follows from Lemma 9 that the only symmetries that fix degree- 3 vertices are colorpreserving reflections and color-preserving rotations of order 3 . Hence the only possible non-trivial stabilizers for degree-3 vertices are:

1. a color-preserving reflection (order 2),

2. a rotation of order 3 (order 3 ) or

3. a rotation of order 3 and three color-preserving reflections (order 6).

Let $\sigma$ denote a counter clockwise rotation of order 3 or 4 for the SSG, let $c$ and $c^{\prime}$ denote the centers of $\sigma$. Let $v_{0}$ be a degree-3 vertex nearest to $c$ but not equal to $c$ and let $v_{i}=\sigma^{i}\left(v_{0}\right)$. Let $\mathcal{C}$ denote the cap determined by $c, v_{0}, v_{1}$ and $v_{2}$ (if $\sigma$ has order 3 ) or by $v_{0}, v_{1}, v_{2}$ and $v_{3}$ (if $\sigma$ has order 4 ). If $\sigma$ has order 3 , we choose the $\Lambda$-coordinate system centered at $v_{0}$ with the center $c$ in the first quadrant having $\Lambda$-coordinates $(x, y)$. Then, inserting a $90^{\circ}$ wedge at $c$, we have $v_{2}=(2 x, 2 y)$ and $v_{3}=(x-y, x+y)$. If $\sigma$ has order 4 , let $(x, y)$ be the $\Lambda$-coordinates of $v_{1}$. Then $v_{2}=(x-y, x+y)$ and $v_{3}=(-y, x)$. By Lemma 8, the rim coordinates of $\mathcal{C}$ are $3(x+y,-x+y)$ and $4(x, y)$, respectively. We may carry out the same analysis for the other cap with $c^{\prime}$ or $v_{0}^{\prime}$ at the origin and $\left(x^{\prime}, y^{\prime}\right)$ as the $\Lambda$-coordinates of $c^{\prime}$ or $v_{1}^{\prime}$ in the bottom cap. Hence the rim coordinates of the botom cap are $3\left(x^{\prime}+y^{\prime},-x^{\prime}+y^{\prime}\right)$ and $4\left(x^{\prime}, y^{\prime}\right)$, respectively. However these rims have the same coordinates. Giving $x^{\prime}=x$ and $y^{\prime}=y$; we conclude that the two caps are isomorphic.

Now suppose that an SSG has been decomposed into two caps and a cylinder and assume that the two caps are congruent under a direct isometry. Relabeling if needed, we may assume that $v_{0}^{\prime}$ is the degree- 3 vertex on the bottom rim that is the image of $v_{0}$ under this direct isometry. The segment joining $v_{0}$ to $v_{0}^{\prime}$ splits the cylinder into a parallelogram and the half-turn about the midpoint of this segment interchanges the caps and the vertices $v_{0}$ and $v_{0}^{\prime}$. Hence this half-turn is a symmetry of the SSG.

If the SSG admits a rotation of order 3 and a rotation of order 4 or two rotations of order 4 with different axes, then it is easy to see that all eight degree-3 vertices lie on 
the same orbit. If there are no reflections the vertex stabilizers have order 3 , the group has order 24 and must be the the direct symmetry group of the cube. In this case, it is natural to visualize the SSG as the cube with the eight degree-3 vertices as corners. It follows from this and the previous argument that remaining possible direct subgroups are:

d. a rotation, $\sigma$ of order 3 and its powers and three half-turns with axes perpendicular to the axis of $\sigma,\left\{\iota, \sigma, \sigma^{2}, \tau_{1}, \tau_{2}, \tau_{3}\right\}$;

e. a rotation, $\sigma$ of order 4 and its powers and four half-turns with axes perpendicular to the axis of $\sigma,\left\{\iota, \sigma, \sigma^{2}, \sigma^{3}, \tau_{1}, \tau_{2}, \tau_{3}, \tau_{4}\right\}$;

f. the 24 element direct symmetry group of the cube.

With these background results, we proceed with with the identification of all possible SSG symmetry groups. For a symmetry group of order 2, we need a color-reversing symmetry of order 2. The only such symmetries listed in Lemma 9 are a color-reversing reflection, a color-reversing half-turn and the color-reversing antipodal map. This accounts for items (i), (ii), the only groups with direct subgroup (a), and (iii), only group equal to (b).

We now turn to the groups of order 4 . The only direct group of order 4 is (c) above and leads directly to group (x). The remaining groups of order 4 are obtained by including two opposite symmetries with the direct subgroup (b) and the composition of these two opposite symmetries must be the half-turn. Hence the only options for a group of order 4 are:

1. one half-turn $\tau$ and two reflections through orthogonal planes containing the axis of $\tau$ and the identity;

2. one half-turn $\tau$, one reflection through the plane orthogonal to the axis of $\tau$, the antipodal map $\alpha$ and the identity;

3. a rotatory-reflection of order 4 and its powers;

In all cases, two of the symmetries must be color-reversing. We consider these possibilities one at a time. There are two ways to select the two symmetries to be color-reversing from the half-turn $\tau$ and the two reflections through orthogonal planes containing the axis of $\tau$. These are listed as items (iv) and (v). There are three ways to select the two symmetries to be color-reversing from the half-turn $\tau$, the reflection through the plane orthogonal to the axis of $\tau$ and the antipodal map $\alpha$. These are listed as items (vi), (vii) and (viii). A rotatory-reflection of order 4 must be color-reversing and results in item (ix).

A symmetry group of order 6 must be group (d) above: if it included opposite symmetries, the index 2 direct subgroup would have order 3 and that is not possible. So the group is $\left\{\iota, \sigma, \sigma^{2}, \tau_{1}, \tau_{2}, \tau_{3}\right\}$. Since $\sigma^{3}=\iota, \sigma$ must be color-preserving and so $\tau_{1}, \tau_{2}, \tau_{3}$ must be color-reversing: group (xi). Moving on to order 8 , either the group equals (e) above or has (c) as its direct subgroup. If it equals (e) either the rotation of order 4 is color-preserving, leading to group (xii), or it is color-reversing, leading to group (xiii). Adding four opposite symmetries to (c) can be done in only two ways: 
1. three reflections with pairwise orthogonal planes of reflection intersecting in the axes of the half-turns and the antipodal map.

2. the odd powers of a rotatory-reflection $\sigma$ of order 4 and two reflections with planes of reflection containing the axis of $\sigma$.

Three distinct versions of the first group listed can occur. Since the reflections generate the group, at least one of them is color-reversing giving the options of one, two or three colorreversing reflections: items (xiv), (xv) and (xvi). There are also three distinct versions of the second group. In this case, the group consists of $\iota, \sigma, \sigma^{2}$, and $\sigma^{3}$, half-turns $\tau_{1}$ and $\tau_{2}$ distinct from the half-turn $\sigma^{2}$, and reflections $\rho_{1}$ and $\rho_{2}$. Since $\sigma^{2}$ is color-preserving and $\tau_{1} \tau_{2}=\sigma^{2}=\rho_{1} \rho_{2}$, our options for four color-reversing symmetries are: (xvii) $\tau_{1}, \tau_{2}, \rho_{1}$, and $\rho_{2} ;$ (xviii) $\sigma, \sigma^{3}, \rho_{1}$, and $\rho_{2} ;(\operatorname{xix}) \sigma, \sigma^{3}, \tau_{1}$, and $\tau_{2}$.

There is no direct group of order 12; hence any group of order 12 must have (d) as its direct subgroup $\left\{\iota, \sigma, \sigma^{2}, \tau_{1}, \tau_{2}, \tau_{3}\right\}$. We note that since $\sigma^{3}=\iota, \sigma$ must be color-preserving and since the half-turns interchange the (differently colored) centers of $\sigma$ they must all be color-reversing. To get the full group we simply add one opposite symmetry and compute its compositions with the symmetries in (d). It is easy to see that at least one of the six added opposite symmetries must be a reflection through a plane containing the axis of $\sigma$. If this plane also contains the axis of one of the half-turns, we get group (xx); if this plane bisects the angle between consecutive half-turn axes, we get group (xxi).

Similarly, there is no direct group of order 16 and all symmetry groups of order 16 must contain (e) as direct subgroup. Again there are just two ways to add the opposite symmetries: one option results in a rotatary-reflection of order 8 , the other is a reflection through a plane perpendicular the axis of the rotation of order 4 . In the first case, the rotatary-reflection having an orbit of length 8 must be color-reversing and group (xxii) is the result. In the second case there are three different ways to choose the color-preserving subgroup: (xxiii), (xxiv) and (xv).

Finally, as already noted, the only possibilities with more than one rotation of order greater than 2 may be identified with the direct and full symmetry groups of the cube. With the red/black bipartite coloring of the vertices of the cube, it is easy to identify the color preserving and color reversing symmetries - item (xxvi) and (xxvii).

\section{Templates for SSGs}

In this section we describe how to construct a template for every SSG and hence for every self-dual spherical grid. Let $\Gamma=\left(V_{b} \cup V_{r} \cup V_{w}, E_{b} \cup E_{r}\right)$ be an SSG. We organize our construction about the symmetry group of $\Gamma$, as listed in Theorem 1 . We will use just four templates: Template 1 for all groups containing three half-turns with pairwise orthogonal axes $\{(x),($ xii), (xiii), (xiv), (xv), (xvi), (xvii), (xviii), (xix), (xxii), (xxiii), (xxiv), (xxv), (xxvi), (xxvii) ; Template 2 for the three groups that include three half-turns with axes in the same plane and no additional half-turns $\{(\mathrm{xi}),(\mathrm{xx}),(\mathrm{xxi})\}$; Template 3 for the groups that include exactly one half-turn $\{$ (iii), (iv), (v), (vi), (vii), (viii), (ix)\}; Template 4 for the two groups that include no half-turns $\{(\mathrm{i}),(\mathrm{ii})\}$. 
Let $\Gamma$ be an SSG with a half-turn $\tau \in \mathcal{A}_{\Gamma}$. Let $c$ and $c^{\prime}$ denote the centers of $\tau$. Let $v_{1}$ be a degree-3 vertex closest to $c$ and let $v_{3}=\tau\left(v_{1}\right)$. Let $v_{0}$ be a degree- 3 vertex next closest to $c$ after $v_{1}$ and $v_{3}$. Then $v_{0}, v_{1}, v_{2}=\tau\left(v_{0}\right)$ and $v_{3}$ form the vertices of a parallelogram $\mathcal{P}_{\tau}$. We call $\mathcal{P}_{\tau}$ "the" parallelogram of $\tau$ at $c$. It is possible that there could be several degree-3 vertices equally close to $c$ at either step of the selection process and a different choice may yield a different parallelogram. Nevertheless no matter how we choose the vertices of $\mathcal{P}_{\tau}$, the remaining equally close vertices lie outside the cap of $\mathcal{P}_{\tau}$. To see this, we observe that, when we copy the cap of $\mathcal{P}_{\tau}$ in $\Lambda$, the entire cap lies in a rectangle that is inscribed in the circle with the segment joining $v_{0}$ and $v_{2}$ as diameter (see Figure 6). The remaining degree-3 vertices must lie on or outside the preimage of that circle in $\Gamma$ and hence outside the cap of $\mathcal{P}_{\tau}$. The remaining four degree-3 vertices must then be the vertices of the parallelogram of $\tau$ at $c^{\prime}$, denoted by $\mathcal{P}_{\tau}^{\prime}$.

Lemma 10. Let $\Gamma$ be an $S S G$ with a half-turn $\tau \in \mathcal{A}_{\Gamma}$. Then $\mathcal{P}_{\tau}$ and $\mathcal{P}_{\tau}^{\prime}$ are directly congruent (congruent under a direct isometry of the sphere) if and only if there exists another half-turn $\tau^{*} \in \mathcal{A}_{\Gamma}$ with axis perpendicular to the axis of $\tau$.

Proof. Let $\Gamma$ be an SSG with a half-turn $\tau \in \mathcal{A}_{\Gamma}$. If a half-turn $\tau^{*}$ exists with axis perpendicular to the axis of $\tau$, it must interchange $\mathcal{P}_{\tau}$ and $\mathcal{P}_{\tau}^{\prime}$. Now suppose that $\mathcal{P}_{\tau}$ and $\mathcal{P}_{\tau}^{\prime}$ are directly congruent and consider the cap and cylinder decomposition of $\Gamma$. Let $v_{0}$ and $v_{2}$ denote the vertices of $\mathcal{P}_{\tau}$ on the top rim and $v_{0}^{\prime}$ and $v_{2}^{\prime}$ denote the vertices of $\mathcal{P}_{\tau}^{\prime}$ on the bottom rim. On easily checks that the segments joining $v_{0}$ to $v_{0}^{\prime}$ and $v_{2}$ to $v_{2}^{\prime}$ partition the cylinder into two congruent parallelograms and that the half-turn $\tau^{*}$ about their centers interchanges the caps and is a symmetry of $\Gamma$.

If follows from Lemma 10 that given an SSG $\Gamma$ with three half-turns $\tau_{1}, \tau_{2}$ and $\tau_{3}$ with perpendicular axes, $\mathcal{P}_{\tau_{i}}$ and $\mathcal{P}_{\tau_{i}}^{\prime}$ are congruent for each index $i$. Now consider the parallelogram $\mathcal{P}_{\tau_{2}}$. Since $\tau_{2}$ rotates $\mathcal{P}_{\tau_{1}}$ into $\mathcal{P}_{\tau_{1}}^{\prime}$, one side of $\mathcal{P}_{\tau_{2}}$ will correspond to one side of $\mathcal{P}_{\tau_{1}}$ and the opposite side of $\mathcal{P}_{\tau_{2}}$ will correspond to a side of $\mathcal{P}_{\tau_{1}}^{\prime}$. A similar conclusion holds of the parallelogram $\mathcal{P}_{\tau_{2}}^{\prime}$ and the parallelograms of $\tau_{3}$. Hence $\Gamma$ may be partitioned into six (three pairs) of parallelograms and each degree- 3 vertex is the vertex of three different parallelograms as pictured in Template 1. The parallelograms of the half-turns fit together to form a distorted cube with the degree- 3 vertices as corners. To identify $\Gamma$ with the template, we select for the origin $v_{0}$, one of the degree- 3 vertices, and one of its neighbors $v_{1}$ to be in the first quadrant; then label the remaining neighbors $v_{2}$ and $v_{3}$ in counterclockwise order. The Basic Conditions ensure this orientation: $x_{1} y_{2}-$ $x_{2} y_{1} \geqslant 0$ implies that $v_{2}$ is counterclockwise from $v_{1} ; x_{2} y_{3}-x_{3} y_{2} \geqslant 0$ implies that $v_{3}$ is counterclockwise from $v_{2} ;-x_{3} x_{1}-y_{3} y_{1} \geqslant 0$ implies that $v_{1}$ is counterclockwise from $v_{3}$. 


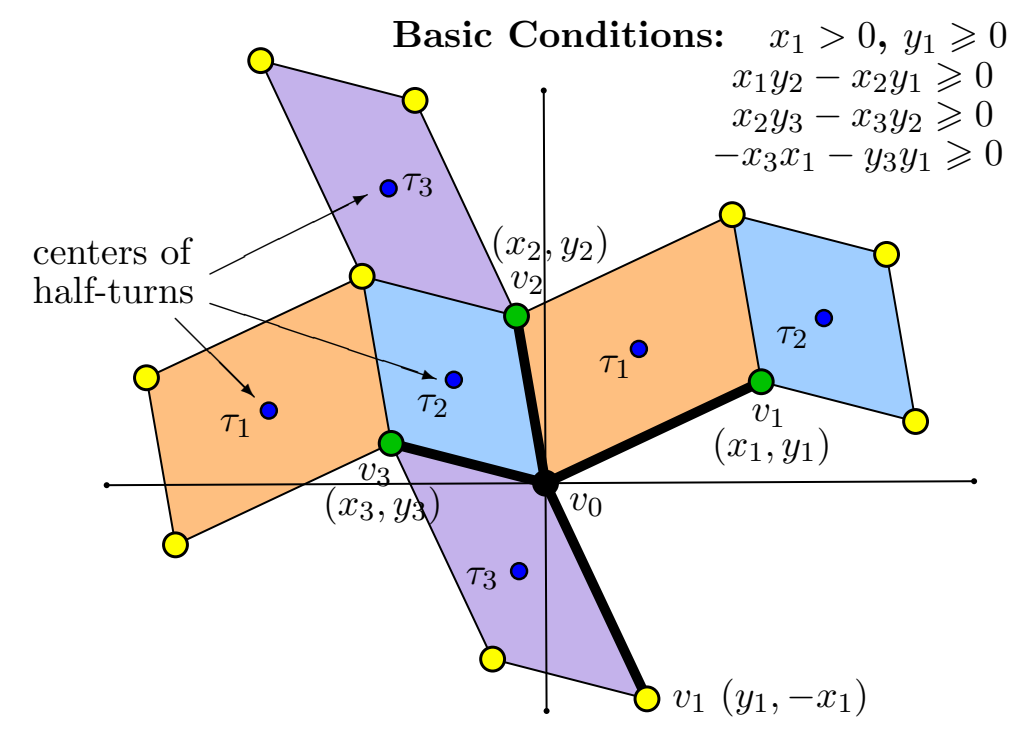

Template \#1: Three half-turns with perpendicular axes.

Lemma 11. Let $\Gamma$ be a $S S G$ where $\mathcal{A}_{\Gamma}$ admits three half-turns with pairwise perpendicular axes. Assume that its degree-3 vertices have been identified with the labeled vertices of Template 1 in $\Lambda$. Assume that $v_{0}$, the degree-3 vertex at the origin, is black (hence the axes are black). Let $\tau_{i}$ denote the half-turn corresponding to the parallelogram defined by $v_{i}$ and $v_{i+1}$ (indices read mod 3). Then:

$i$. The number of vertices of the underlying self-dual $S G$ is given by

$$
\frac{x_{1} y_{2}-x_{2} y_{1}+x_{2} y_{3}-x_{3} y_{2}-x_{3} x_{1}-y_{3} y_{1}}{2}+1
$$

ii. $x_{i} \equiv y_{i}(\bmod 2)$ for all $(i)$ and $x_{i} \equiv 1(\bmod 2)$ for at least one index.

iii. If $x_{i} \equiv x_{i+1}(\bmod 2)$ (indices read mod 3), then $\tau_{i}$ is color-preserving, otherwise $\tau_{i}$ is color-reversing.

iv. If $\mathcal{A}_{\Gamma}$ admits an orientation reversing symmetry, $\Gamma$ may be oriented so that one of the following conditions holds:

(a) $x_{i}=y_{i}$ for all $i$ (all edges of the parallelepiped have SSG-coordinates of the form $(s, s))$;

(b) $x_{1}=y_{1}$ and $y_{2}=y_{3}=0$ (one edge of the parallelepiped has SSG-coordinates of the form $(s, s)$, the remaining two have $S S G$-coordinates of the form $(s))$;

(c) $x_{2}=-x_{1}, y_{2}=y_{1}$ and $y_{3}=0$ (one edge of the parallelepiped has $S S G$ coordinates of the form $(s)$, the remaining two have $S S G$-coordinates of the form $(s, t)$ and $(t, s))$

$v$. $v_{0}$ is the center of a rotation of order 3 if and only if $x_{2}=-y_{1}, y_{2}=x_{1}, x_{3}=-x_{1}$ and $y_{3}=-y_{1}$ (all edge of the parallelepiped at $v_{0}$ have the same SSG-coordinates). 
vi. If $\Gamma$ admits a rotation $\sigma$ of order 4 , we assume that the center of $\tau_{1}$ is actually the center of $\sigma$ with $\tau_{1}=\sigma^{2}$. In this case, $x_{2}=-y_{1}$ and $y_{2}=x_{1}$. In fact, the parallelograms of $\tau_{1}$ are squares; all edges of these parallelograms have the same SSG-coordinates $(p, q)$. Furthermore, if $p$ and $q$ are even $\sigma$ is color-preserving, if $p$ and $q$ are odd $\sigma$ is color-reversing. In either case, $\tau_{1}=\sigma^{2}$ is color-preserving.

Proof.

i. $x_{1} y_{2}-x_{2} y_{1}$ is the area of the parallelogram that is defined by the vectors $\left(x_{1}, y_{1}\right)$ and $\left(x_{2}, y_{2}\right)$ with similar formulas for the areas remaining parallelograms at the origin. Adding these these areas and doubling gives the total area. Then dividing by 4 and adding 1 gives the number of vertices of the underlying self-dual SG by Lemma 7 .

ii. Since the origin is a black vertex, $v_{i}$ will be black if and only if $x_{i} \equiv y_{i} \equiv 0(\bmod 2)$ and red if and only if $x_{i} \equiv y_{i} \equiv 1(\bmod 2)$. If $x_{i} \equiv y_{i} \equiv 0(\bmod 2)$ for all $i$, then all degree- 3 vertices would be black.

iii. If $x_{i} \equiv x_{i+1}(\bmod 2)$, then $v_{i}$ and $v_{i+1}$ are the same color and $\tau_{i}$ is color-preserving. If $x_{i} \not \equiv x_{i+1}(\bmod 2)$, then $v_{i}$ and $v_{i+1}$ are different colors and $\tau_{i}$ is color-reversing.

iv. Let $\mathcal{P}_{i} \cong \mathcal{P}_{i}^{\prime}$ denote the parallelograms of $\tau_{i}$. We note first that under any opposite symmetry $\mathcal{P}_{1}$ is mapped onto itself, onto $\mathcal{P}_{1}^{\prime}$ or onto one of the other four parallelograms. It follows that either all parallelograms have reflective symmetry or one pair has reflective symmetry and the other two pairs are reflections of one another. We may assume that $\mathcal{P}_{1}$ has reflective symmetry. Thus $\mathcal{P}_{1}$ admits two reflections. Either the axes of the reflections of $\mathcal{P}_{1}$ are perpendicular to its sides and $\mathcal{P}_{1}$ is a rectangle or the axes of the reflections of $\mathcal{P}_{1}$ are its diagonals. In the first case the SSG-coordinates of its sides must have the form $(p, p)$ and the axis of reflection must carry on through the sides of the neighboring parallelograms forcing all sides of the parallelopiped to have SSG-coordinates of the form $(p, p)$; option (a). There are just two ways that the axes of the reflections of $\mathcal{P}_{1}$ can be its diagonals giving options (b) and (c).

v. If $v_{0}$ is the center of a rotation of order 3 the vector to $v_{2}$ is the image of the vector to $v_{1}$ under the $90^{\circ}$ counterclockwise rotation: hence $\left(x_{2}, y_{2}\right)=\left(-y_{1}, x_{1}\right)$; similarly, $\left(x_{3}, y_{3}\right)=\left(-x_{1},-y_{1}\right)$.

vi. By theorem 1 if $\sigma$ has order $4, \sigma^{2}$ is one of a set of three half-turns with pairwise orthogonal axes. Since the parallelograms of $\sigma^{2}$ are mapped onto themselves under a $90^{\circ}$ rotation they are squares and the edges at $v_{0}$ are perpendicular. Hence $\left(x_{2}, y_{2}\right)=$ $\left(-y_{1}, x_{1}\right)$. Adjacent degree- 3 vertices around this square face will have the same color when $x_{1}$ and $y_{1}$ are even and they will alternate in color when $x_{1}$ and $y_{1}$ are odd. 
We now consider each of the symmetry groups described by Template 1 .

$\mathcal{A}_{\Gamma}$ is of Type (xxvii). Since the vertex stabilizers have order 6 , we have a rotation of order 3 about the origin giving $x_{2}=-y_{1}, y_{2}=x_{1}, x_{3}=-x_{1}$ and $y_{3}=-y_{1}$. We also have a reflection fixing the origin, hence we have just one parameter $p: x_{1}=y_{1}=y_{2}=p$ and $x_{2}=x_{3}=y_{3}=-p$. To avoid all vertices having the same color, $p$ must be a positive odd integer. The number of vertices in $\Gamma_{b}$ is $3 p^{2}+1$ and taking $p=1$ gives the smallest example: the tetrahedron.

$\mathcal{A}_{\Gamma}$ is of Type (xxvi). Since the vertex stabilizers have order 3 , we have a rotation of order 3 about the origin giving $x_{2}=-y_{1}, y_{2}=x_{1}, x_{3}=-x_{1}$ and $y_{3}=-y_{1}$. Since we also have no orientation reversing symmetries, we have two parameters $p$ and $q$ : $x_{1}=y_{2}=$ $-x_{3}=p$ and $-x_{2}=-y_{3}=y_{1}=q$. Furthermore, $p$ and $q$ must be distinct positive odd integers. We then have that the number of vertices of $\Gamma_{b}$ is $\frac{3}{2}\left(p^{2}+q^{2}\right)+1$. Hence taking $p=1$ and $q=3$ gives the smallest example with 16 vertices.
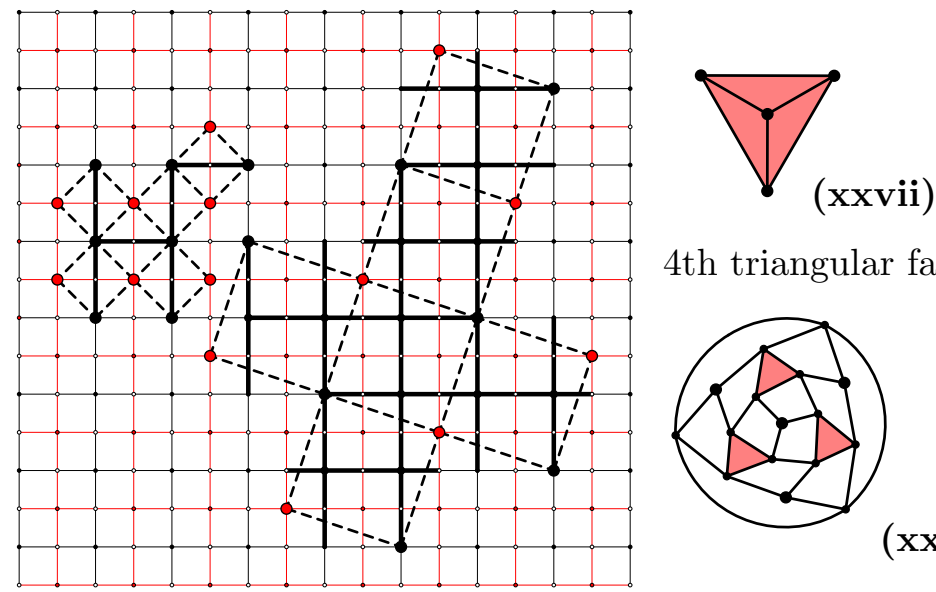

4th triangular face outside

Figure 8: Smallest Examples (xxvii) \& (xxvi)

For the next three cases, we have a single rotation $\sigma$ of order 4 with four reflections through planes containing the axis of $\sigma$. It follows that the SSG-coordinates of the sides of the square face must be all of the form $(p)$ or all of the form $(p, p)$. The reflection through the plane containing a degree- 3 vertex interchanges the two edges of the square face and fixes the third edge leaving that vertex. We conclude that the three edges at a degree-3 vertex are all of the form $(p)$ or all of the form $(p, p)$. If they were all of the form $(p)$ all degree- 3 vertices would have the same color. Hence, in these cases $x_{1}=y_{1}=\left(-x_{2}\right)=y_{2}=p$ and $x_{3}=y_{3}=-q$. Parameters $p$ and $q$ must be distinct positive integers to avoid a rotation of order 3 , and at least one is odd to ensure that half of the degree- 3 vertices are of each color. The number of vertices is $p^{2}+2 p q+1$. Let $\rho$ denote the reflection through the plane perpendicular to the axis $\sigma$. Then the options are:

$\mathcal{A}_{\Gamma}$ is of Type (xxv). $\sigma$ and $\rho$ are both color-reversing: $p$ and $q$ odd. To get the smallest example, let $p=1$ and $q=3$ giving $\left|V_{b}\right|=8$.

$\mathcal{A}_{\Gamma}$ is of Type (xxiv). $\sigma$ color-reversing and $\rho$ color-preserving: $p$ odd and $q$ even. To 
get the smallest example, let $p=1$ and $q=2$ giving $\left|V_{b}\right|=6$.

$\mathcal{A}_{\Gamma}$ is of Type (xxiii). $\sigma$ color-preserving and $\rho$ color-reversing: $p$ even and $q$ odd. To get the smallest example, let $p=2$ and $q=1$ giving $\left|V_{b}\right|=9$.

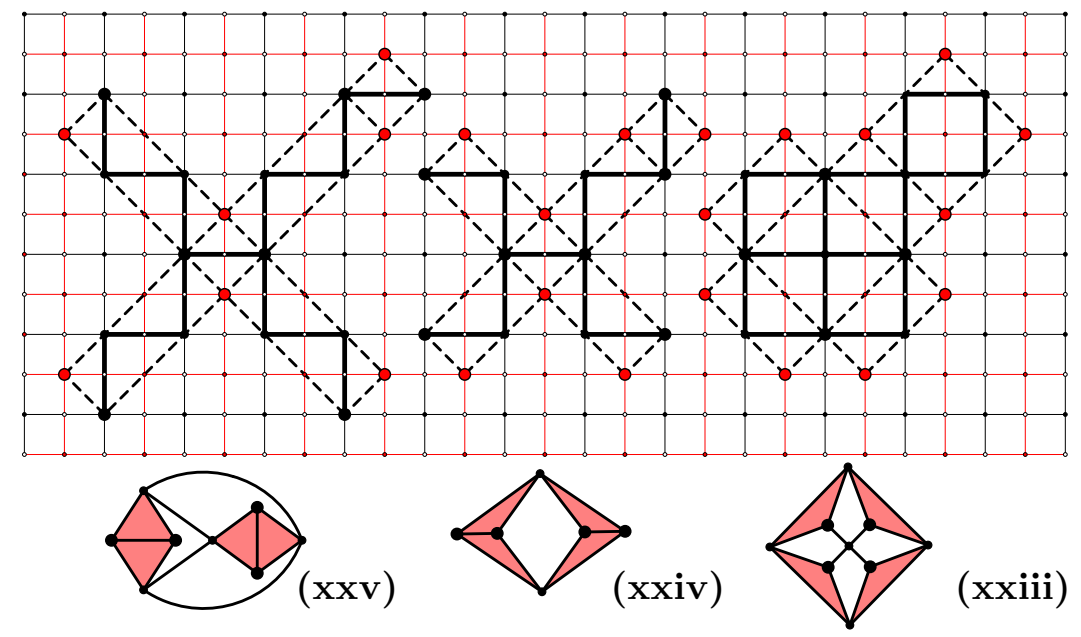

Figure 9: Smallest Examples (xxv), (xxiv) \& (xxiii)

$\mathcal{A}_{\Gamma}$ is of Type (xxii). Since we have a color-reversing rotatory-reflection $\phi$ of order 8 , we have that the rotation of order $4, \phi^{2}$, and the half-turn $\phi^{4}=\tau_{1}$ are both color-preserving. It follows that the $\tau_{1}$ parallelogram is a square. Since there are also reflections through planes containing the axis of $\phi$, the edge SSG-coordinates are of the form $(r)$ or $(r, r)$ where $r$ is even. Now the bottom parallelogram is congruent to the top parallelogram but is rotated $45^{\circ}$. Since the plane of reflection of $\phi$ is perpendicular to the axis of $\phi$, we conclude that $v_{0}$ (the origin), $v_{2}$ and $v_{3}$ form an isosceles triangle with the base vertices $v_{0}$ and $v_{2}$ having the same color and $v_{3}$ having a different color. This can only happen if $y_{2}=x_{1}=2 p, x_{2}=y_{1}=0$ and $x_{3}=-q$ and $y_{3}=p$ where both $p$ and $q$ are odd. Here we have that the number of vertices of $\Gamma_{b}$ is $2 p^{2}+2 p q+1$. Hence taking $p=1$ and $q=1$ gives the smallest example with 5 vertices.

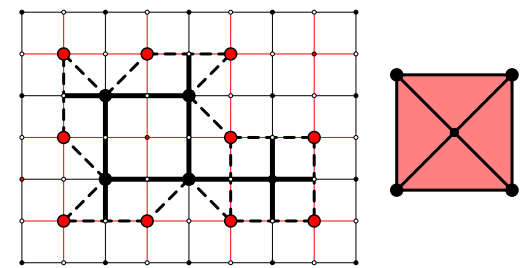

Figure 10: Smallest Example (xxii)

Cases of Types (xxi) and (xx) are represented by Template \#2 and will be considered later. We move down to groups of order 8. The next three cases (xix), (xviii) and (xvii) all have a rotatory-reflection $\sigma$ of order 4 and we assume that $\sigma^{2}=\tau_{1}$ in the template. They also have reflections through perpendicular planes containing the axis of $\sigma$. Thus each the 
two parallelograms for $\tau_{1}$ must have two perpendicular axes of symmetry. Furthermore, two parallelograms for $\tau_{1}$ must be perpendicular to one another when viewed along the axis of $\tau_{1}$. There are three different ways to accomplish this:

a. Let the axes of symmetry be the diagonals of the parallelogram, that is sides of the parallelogram have SSG-coordinates $(p, q),(q, p),(p, q),(q, p)$ in cyclic order. Specifically: $x_{1}=y_{2}=p, x_{2}=y_{1}=q, x_{3}=-2 r, y_{3}=0$, where $p>q>0, r>0$ and $p, q$ odd (without this condition all vertices would be the same color). We have $\left|V_{b}\right|=\frac{p^{2}-q^{2}}{2}+2 p r+1$. Since $\sigma\left(v_{2}\right)=v_{3}, \sigma$ is color-reversing. Since $p$ and $q$ are odd, both reflections through perpendicular planes containing the axis of $\sigma$ are color-preserving. So this construction yields SSGs with symmetry group (xix). The smallest example of this type has $p=3$ and $q=r=1$ giving $\left|V_{b}\right|=11$.

If the axes of symmetry of the parallelogram are not the diagonals, the parallelogram must be a rectangle with side SSG-coordinates of the form $(p, p)$ or $(p)$ leading to the next two cases.

b. $x_{1}=2 p, y_{2}=2 q, y_{1}=x_{2}=0, x_{3}=-r$ and $y_{3}=p+q$, where $p>q>0, r>0, r$ and $p+q$ odd. To avoid an additional reflection, we must require the $r \neq p-q$. We have $\left|V_{b}\right|=2 p q+r(p+q)+1$. Since the vertices of the top and bottom rectangles have different colors, $\sigma$ is color-reversing. But the reflections through perpendicular planes containing the axis of $\sigma$ are color-preserving. So this construction also yields SSGs with symmetry group (xix). The smallest example of this type has $p=2$, $q=1$ and $r=3$ giving $\left|V_{b}\right|=14$.

c. $x_{1}=y_{1}=p,-x_{2}=y_{2}=q, x_{3}=-\frac{p+q}{2}-r$ and $y_{3}=\frac{p+q}{2}-r$, where $p>q>0, r>0$ and $p+q$ even. To avoid an additional reflection, we must require the $r \neq \frac{p-q}{2}$, $r \neq \frac{p+q}{2}$ and $r \neq \frac{p+3 q}{2}$. We have $\left|V_{b}\right|=p q+r(p+q)+1$. Since $\sigma\left(v_{1}\right)=v_{3}, \sigma$ is color-reversing when $\frac{p+q}{2}-r$ is odd and color-preserving when $\frac{p+q}{2}-r$ is even. The reflections through perpendicular planes containing the axis of $\sigma$ are colorreversing when $p$ and $q$ are odd and preserving when $p$ and $q$ are even. There are four possibilities:

(1) $\frac{p+q}{2}-r$ odd, $p$ and $q$ odd, symmetry group (xviii), smallest example $p=7$ and $q=r=1$ giving $\left|V_{b}\right|=16$ or $p=3, q=1$ and $r=3$ giving $\left|V_{b}\right|=16$

(2) $\frac{p+q}{2}-r$ odd, $p$ and $q$ even, symmetry group (xix), smallest example $p=6$, $q=2$ and $r=1$ giving $\left|V_{b}\right|=21$.

(3) $\frac{p+q}{2}-r$ even, $p$ and $q$ odd, symmetry group (xvii), smallest example $p=5$ and $q=r=1$ giving $\left|V_{b}\right|=12$.

(4) $\frac{p+q}{2}-r$ even, $p$ and $q$ even, would produce an SSG with all degree-3 vertices of the same color.

$\mathcal{A}_{\Gamma}$ is of Type (xix). Since $\sigma$ is color-reversing and the reflections are color-preserving, each of the three configurations is possible. The smallest possibility for (a) has $\left|V_{b}\right|=11$, the smallest for (b) has 14 vertices and the smallest for (c) has 21 vertices. 
$\mathcal{A}_{\Gamma}$ is of Type (xviii). Since $\sigma$ and the reflections are color-reversing, only configuration (c) is possible and the smallest example has $\left|V_{b}\right|=16$.

$\mathcal{A}_{\Gamma}$ is of Type (xvii). Since $\sigma$ is color-preserving and the reflections are color-reversing only configuration (c) is possible and the smallest example has $\left|V_{b}\right|=12$.

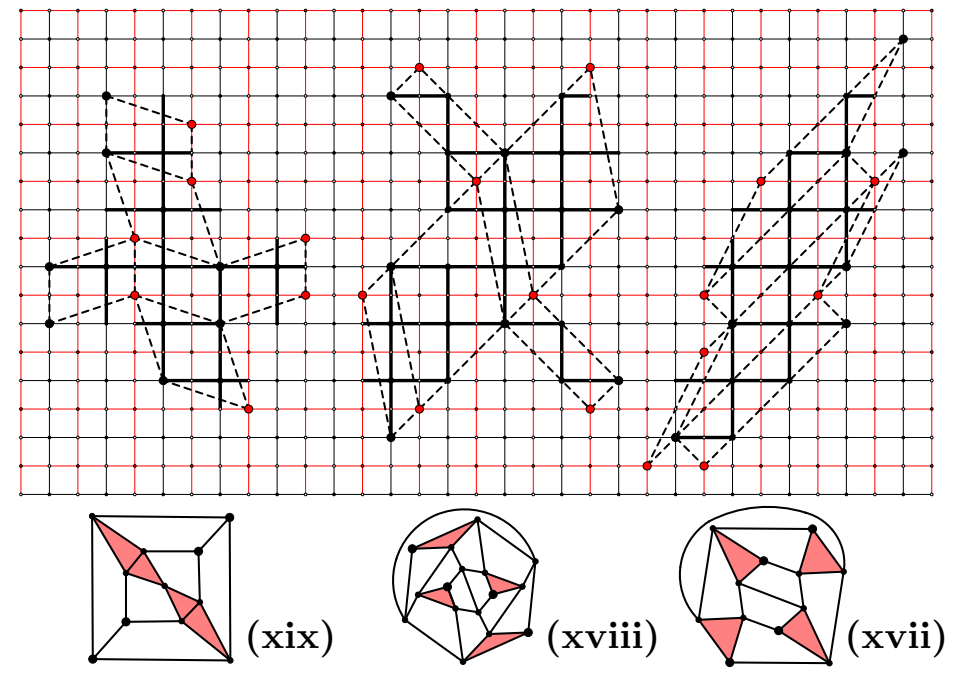

Figure 11: Smallest Examples (xix), (xviii) \& (xvii)

The next three groups have three reflections with pairwise orthogonal equators. Let $\rho_{1}$ be one of the color-reversing reflections and let $\tau_{1}$ be the composition of the other two. Then the plane of $\rho_{1}$ is perpendicular to the axis of $\tau_{1}$. Since $\rho_{1}$ is color-reversing its equator is a $45^{\circ}$ line. Hence the rim of $\tau_{1}$ must be a $45^{\circ}$ line.

The options for the top parallelogram of $\tau_{1}$ are the same three structures we discussed for the rotatory-reflection of order 4 . In the first two cases (a) and (b) above, the rim of $\tau_{1}$ is a horizontal line. Hence case (c) must hold: $x_{1}=y_{1}=p$ and $-x_{2}=y_{2}=q$, $p>q>0$. The vertex $\left(x_{3}, y_{3}\right)$ is the reflected image of the degree- 3 vertex at the origin. Hence $x_{3}=-r$ and $y_{3}=-r$, where $r$ is odd. To avoid additional symmetries, $r$ is distinct from $p$ and $q$. The reflections perpendicular to the edges with SSG-coordinates $(p, p)$ and $(q, q)$ will be color-preserving when $p$ (respectively $q$ ) is even and color-reversing when it is odd. We have $\left|V_{b}\right|=p q+p r+q r+1$.

$\mathcal{A}_{\Gamma}$ is of Type (xvi). One color-reversing reflection, so exactly one of $p, q$ and $r$ is odd. The smallest example has $p=4, q=2$ and $r=1$ giving $\left|V_{b}\right|=15$.

$\mathcal{A}_{\Gamma}$ is of Type (xv). Two color-reversing reflections, so exactly two of $p, q$ and $r$ are odd. The smallest example has $p=3, q=2$ and $r=1$ giving $\left|V_{b}\right|=12$.

$\mathcal{A}_{\Gamma}$ is of Type (xiv). Three color-reversing reflections, so all of $p, q$ and $r$ are odd. The smallest example has $p=5, q=3$ and $r=1$ giving $\left|V_{b}\right|=24$.

The next two cases consist of a rotation $\sigma$ of order 4 and four half-turns with axes perpendicular to the axis of $\sigma$ and no reflections. Hence we have $x_{1}=y_{2}=p$ and $y_{1}=-x_{2}=q$ to give the square with its center as the center of the rotation of order 


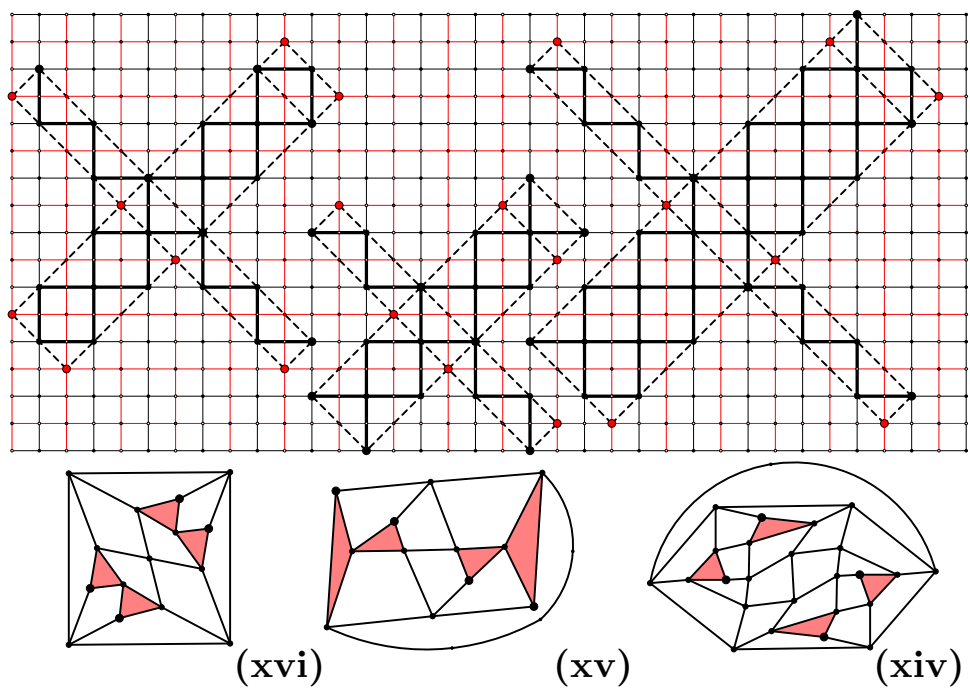

Figure 12: Smallest Examples (xvi), (xv) \& (xiv)

4. The remaining parameters $x_{3}$ and $y_{3}$ must satisfy the basic conditions, which become $\frac{p}{q} \geqslant \frac{y_{3}}{-x_{3}}$. Furthermore they must be different from $(-p,-q)$ to avoid a rotation of order 3. If $p=q$ or $q=0, x_{3}$ and $y_{3}$ must be chosen to avoid a reflection or a rotatory-reflection of order 8 (see xxii). If $p$ and $q$ are odd, $\sigma$ is color-reversing; if $p$ and $q$ are even, $\sigma$ is color-preserving and $x_{3}$ and $y_{3}$ must be odd. We have $\left|V_{b}\right|=\frac{p^{2}+q^{2}}{2}-p x_{3}-q y_{3}+1$.

$\mathcal{A}_{\Gamma}$ is of Type (xiii). One color-reversing rotation $\sigma$ of order 4 ; so $p$ and $q$ are odd. The smallest example has $p=3, q=1 x_{3}=y_{3}=-1$ for $\left|V_{b}\right|=10$.

$\mathcal{A}_{\Gamma}$ is of Type (xii). One color-preserving rotation $\sigma$ of order 4 ; so $p$ and $q$ are even, $x_{3}$ and $y_{3}$ odd. The smallest example has $p=4, q=0 x_{3}=-1, y_{3}=1$ for $\left|V_{b}\right|=13$.

The very last case to consider using Template \#1 is:

$\mathcal{A}_{\Gamma}$ is of Type (x). Just three half-turns with pairwise perpendicular axes. In addition to the basic conditions on the parameters they must be chosen to avoid any reflections. Since two of the half-turns must be color-reversing, we must avoid having all six parameters even or all six odd. The smallest example has $x_{1}=3, y_{1}=1, x_{2}=0, y_{2}=2, x_{3}=-2$ and $y_{3}=2$ for $\left|V_{b}\right|=8$.

We need a different template for the SSGs with automorphism groups that contain just one rotation of order 3 and hence three half-turns; all axes in a plane. To construct Template \#2, let $\Gamma$ be such an SSG and let $v_{0}$ be a center of the (counterclockwise) rotation $\phi$ of order 3 and let $v_{1}$ be a nearest degree- 3 vertex. Then $v_{1}, \phi\left(v_{1}\right)$ and $\phi^{2}\left(v_{1}\right)$ have all the same the SSG coordinates. We plot the corresponding points in $\Lambda$ with $v_{0}$ at the origin, $v_{1}$ in the first quadrant and $\phi\left(v_{1}\right)$ and $\phi^{2}\left(v_{1}\right)$ the $90^{\circ}$ and $180^{\circ}$ counterclockwise rotations of $v_{1}$ about the origin (denoted by $v_{3}$ and $v_{4}$ in the template). Since $\phi$ also rotates the centers of the half-turns, they are also evenly spaced about $v_{0}$. We denote by $\tau_{1}$ the half-turn with center $c_{1}$ nearest to $v_{1}$ in the counterclockwise direction. The centers $c_{2}$ and $c_{3}$ are the images of $c_{1}$ under $\phi$ and $\phi^{2}$. Finally, let $v_{2}$ denote the image of $v_{1}$ under $\tau_{1}$. 


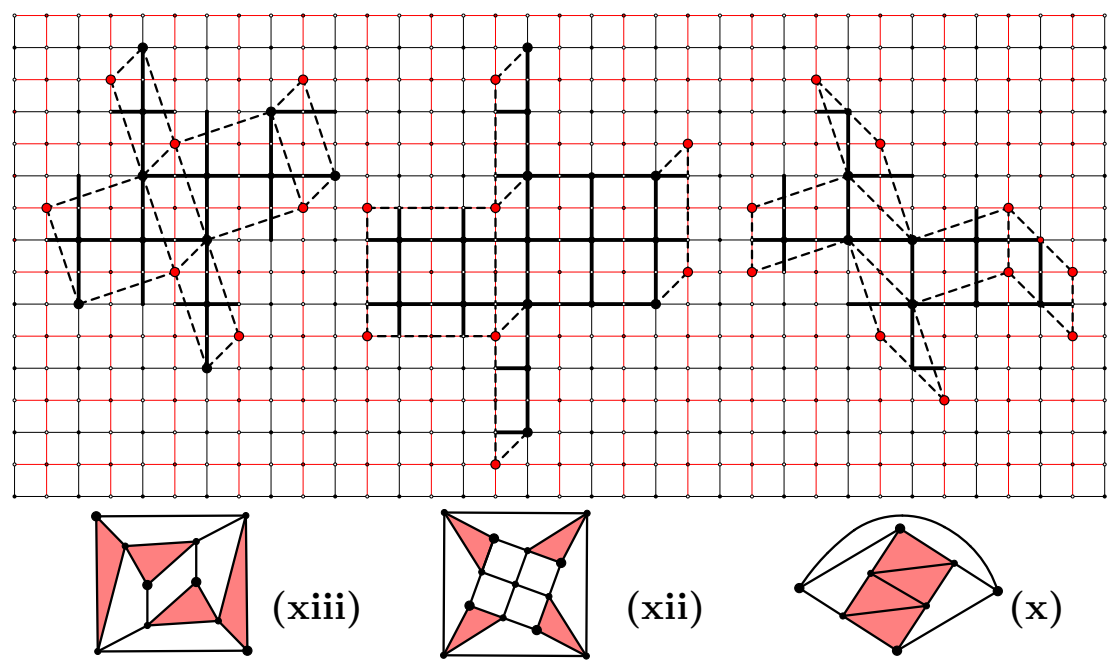

Figure 13: Smallest Examples (xiii), (xii) \& (x)

The quadrilateral $v_{0}, v_{1}, v_{2}, v_{3}$ is then our basic unit: the three copies of this quadrilateral under $\phi$ surround $v_{0}$ and they dovetail with the three copies surrounding $v_{0}^{\prime}$ to complete the template.

Basic Conditions: $x_{1}>0, y_{1} \geqslant 0$; one pair $\left(x_{1}, y_{1}\right),\left(x_{2}, y_{2}\right)$ even, the other odd; $x_{1} y_{2}-x_{2} y_{1} \geqslant 0, x_{2} x_{1}+y_{1} y_{2} \geqslant 0$ and $\left(x_{2}, y_{2}\right) \neq\left(x_{1}-y_{1}, x_{1}+y_{1}\right)$

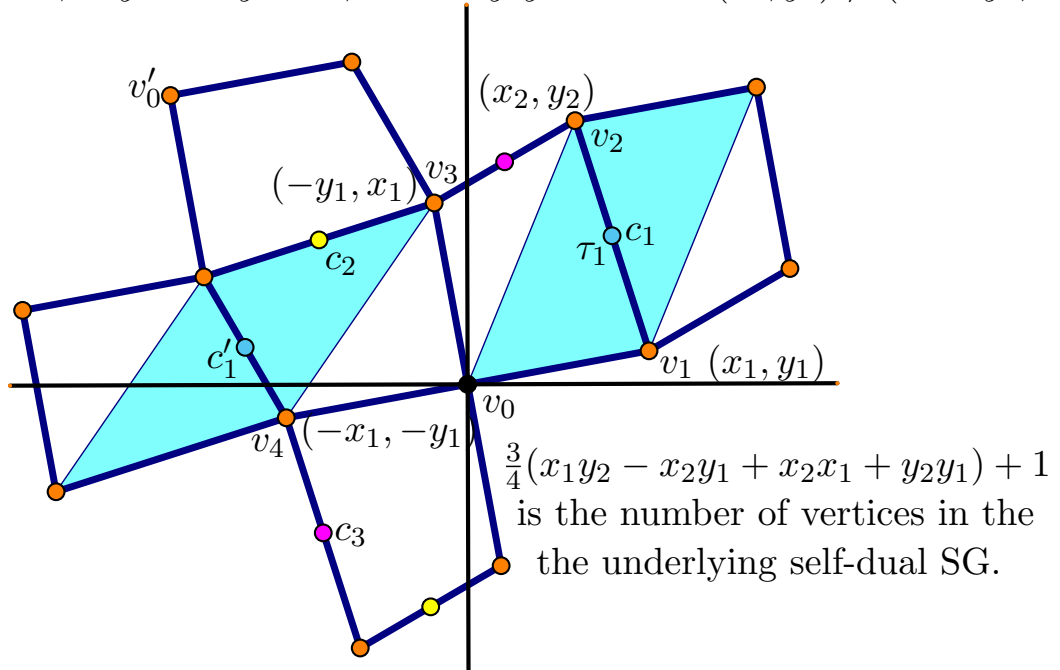

Template \#2: Three half-turns; all axes in a plane perpendicular to the axis of a rotation of order 3.

To avoid additional symmetries in Template \#2, we must ensure that the two parallelograms corresponding to the half-turn $\tau_{i}$ are not congruent. The parallelograms for $\tau_{1}$ are highlighted in the template. The condition that these parallelograms are not congruent is easily seen to be equivalent to the condition that the quadrilateral $v_{0}, v_{1}, v_{2}, v_{3}$ is not a square and this, in turn, is equivalent to the condition $\left(x_{2}, y_{2}\right) \neq\left(x_{1}-y_{1}, x_{1}+y_{1}\right)$. To compute the area of the quadrilateral, we note that $\frac{1}{2}\left(x_{1} y_{2}-x_{2} y_{1}\right)$ is the area of the triangle with vertices $v_{0}, v_{1}$ and $v_{2}$. Similarly $\frac{1}{2}\left(x_{2} x_{1}+y_{1} y_{2}\right)$ is the area of the triangle 
with vertices $v_{0}, v_{2}$ and $v_{3}$. Their sum is then the area of the basic quadrilateral and six times their sum or $3\left(x_{1} y_{2}-x_{2} y_{1}+x_{2} x_{1}+y_{2} y_{1}\right)$ is the total area.

$\mathcal{A}_{\Gamma}$ is of Type (xxi). In order to accommodate the rotatory-reflection and the reflections through the axis of $\phi$, we must have either $y_{1}=0$ and $x_{2}=y_{2}$ or $x_{1}=y_{1}$ and $x_{2}=0$. The smallest example is given by $x_{1}=y_{1}=1, x_{2}=0$ and $y_{2}=4$ for $\left|V_{b}\right|=7$.

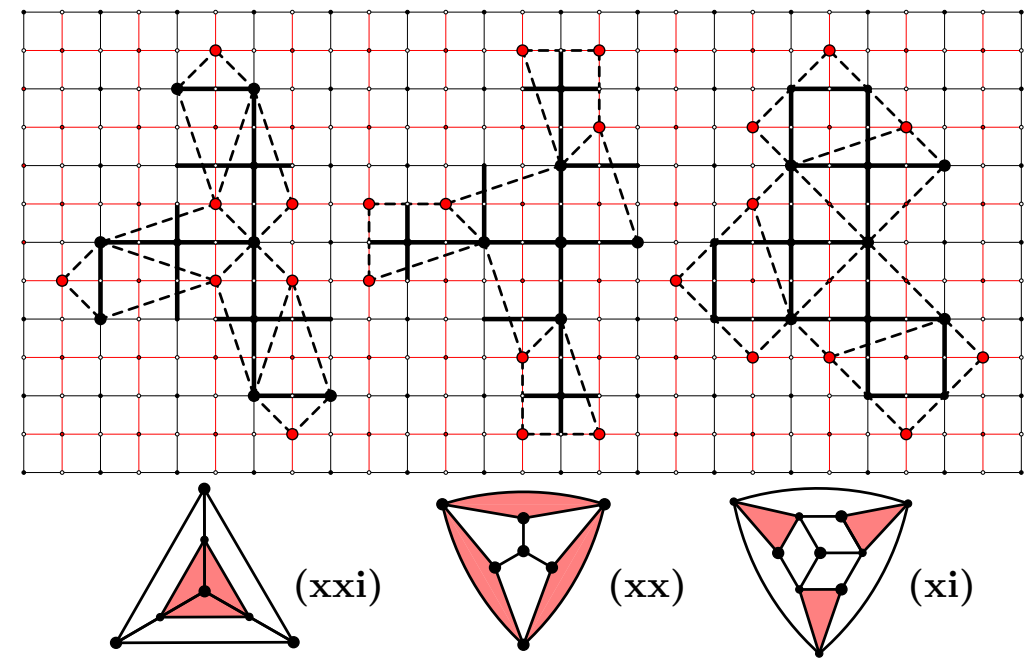

Figure 14: Smallest Examples (xxi), (xx) \& (xi)

$\mathcal{A}_{\Gamma}$ is of Type $(\mathbf{x x})$. In order to accommodate the reflection through the plane containing the axes of the half-turns and the reflections through the axis of $\phi$, we must have $y_{1}=0$ and $y_{2}=x_{2}+x_{1}$. The smallest example is given by $x_{1}=2, y_{1}=0, x_{2}=1$ and $y_{2}=3$ for $\left|V_{b}\right|=7$.

$\mathcal{A}_{\Gamma}$ is of Type (xi). Avoiding additional reflections, the smallest example is given by $x_{1}=2, y_{1}=2, x_{2}=1$ and $y_{2}=3$ for $\left|V_{b}\right|=10$.

In constructing these smallest examples, the main problem is avoiding additional symmetries. Adding one additional symmetry will double the group size. Hence for large groups, there will be many additional symmetries and their presence will be easily detected and avoided. However, with the small groups that we encounter next, additional are easy to miss; in particular color-preserving reflections with equators containing some or all of the degree- 3 vertices are very easy to miss. Template \#3 covers the seven groups that admit exactly one half-turn. It and Template \#4 will use the cap and cylinder decomposition introduced in Section 4. In view of Lemma 10, we must ensure that the two parallelograms of this half-turn are not congruent. The listed Basic Conditions insure that this is the case. 


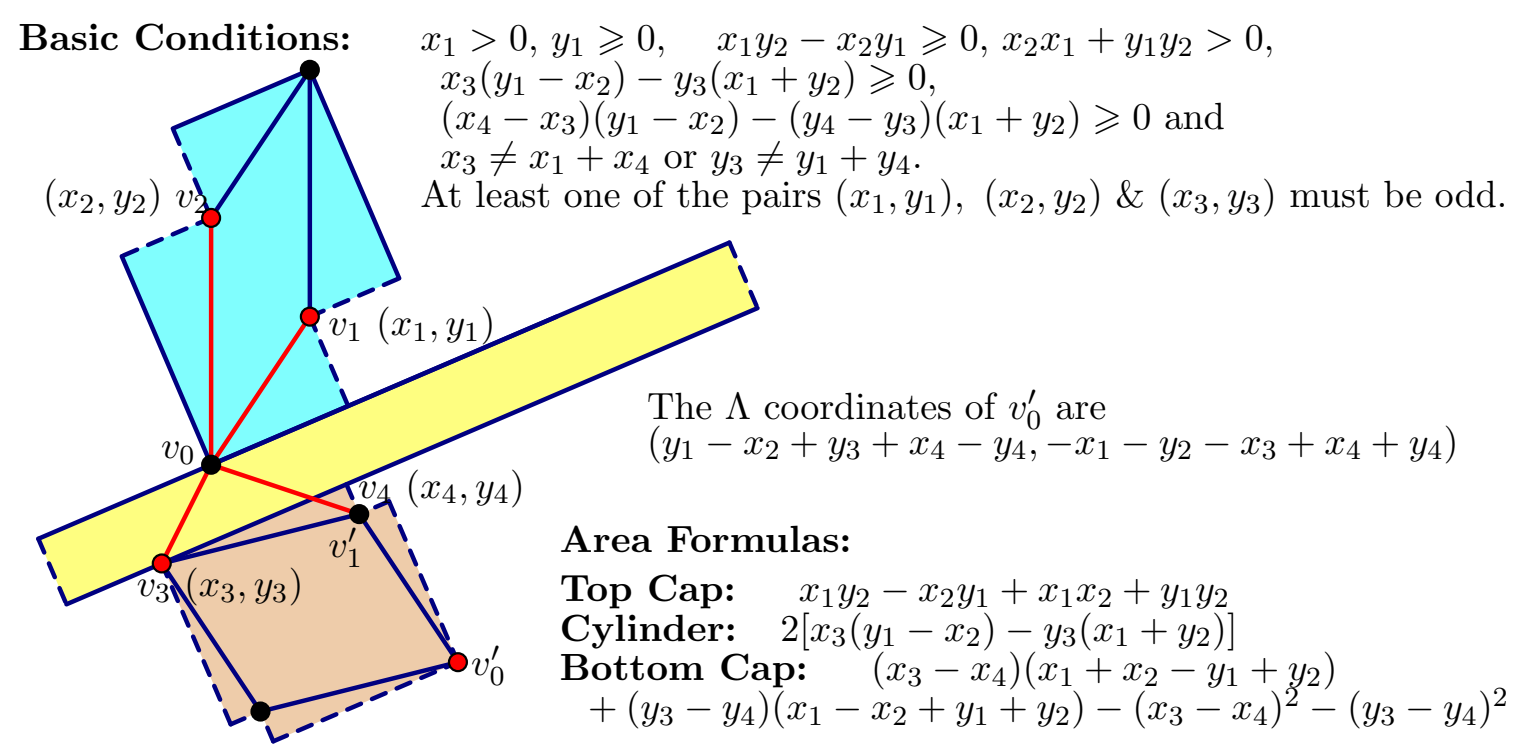

Template \#3: One half-turn.

Lemma 12. Let $\Gamma$ be an SSG that admits only one half-turn, $\tau$. Then it may be orientated as in Template \#3 and the basic conditions listed must be satisfied.

a. For groups (iv), (v), (vi), (vii) and (ix), the rims must be $45^{\circ}$ segments with the axis of reflection the centerline of the cylinder parallel to the rims.

b. For groups (iv) and (v) the parallelograms must be rectangles that are non-congruent with $45^{\circ}$ sides so that the midpoints of the $v_{0}, v_{1}$ segment and the $v_{3}, v_{4}$ segment must match up across the cylinder: $v_{1}=(p, p), v_{2}=(-q, q), v_{3}=\left(\frac{p-s}{2}+r, \frac{p-s}{2}-r\right)$ and $v_{4}=\left(\frac{p+s}{2}+r, \frac{p+s}{2}-r\right)$. For these groups the number of vertices in the underlying $S G$ is $\frac{1}{2}\left(p q+(s+2 r)(p+q)-s^{2}\right)+1$, where $(r,-r)$ is the width of the rim and $(s, s)$ is the width of the bottom rectangle and $p \geqslant q>0, s>0, s \neq p, q$ and $s \geqslant \frac{p+q}{2}$.

c. For groups (vi), (vii), (viii) and (ix) the parallelograms must have no reflective symmetry and be the reflections of one another. For groups (vi), (vii) and (ix) the number of vertices in the underlying $S G$ is $\frac{1}{2}\left(y_{1}^{2}-x_{1}^{2}-2 * y_{1} x_{2}\right)+r\left(y_{1}-x_{2}\right)+1$, where $(r,-r)$ is the width of the rim. An SSG with group (viii) may have a $45^{\circ}$ equator of reflection or it may have an equator of reflection through some of the degree-3 vertices.

Finally, we note that $\tau$ will be color-preserving when the pairs $\left(x_{1}, y_{1}\right)$ and $\left(x_{2}, y_{2}\right)$ have the same parity and color-reversing when they have different parity. When $x_{3}$ above is even any reflection or rotatory-reflection through the cylinder is color-preserving; when $x_{3}$ above is odd any such reflection or rotatory-reflection is color-reversing.

Proof. We may assume that the top parallelogram for $\tau$ has a vertex $v_{0}$ on the long diagonal at the origin and $v_{1}$ in the first quadrant $\left[x_{1}>0, y_{1} \geqslant 0\right]$. The vertex $v_{2}$ is counterclockwise from $v_{1}\left[x_{1} y_{2}-x_{2} y_{1} \geqslant 0\right]$ with $\angle v_{2} v_{0} v_{1}$ less than $90^{\circ}\left[x_{2} x_{1}+y_{1} y_{2}>0\right]$. 
From Lemma 8 , the rim coordinates are $2\left(x_{1}+y_{2}, y_{1}-x_{2}\right)$. Hence the direction to vertex $v_{3}$ of the bottom parallelogram on the bottom rim must be clockwise from the top rim $\left[x_{3}\left(y_{1}-x_{2}\right)-y_{3}\left(x_{1}+y_{2}\right) \geqslant 0\right]$. Finally $v_{4}$, vertex on the bottom parallelogram adjacent to $v_{3}$ must be on or below the bottom rim $\left[\left(x_{4}-x_{3}\right)\left(y_{1}-x_{2}\right)-\left(y_{4}-y_{3}\right)\left(x_{1}+y_{2}\right) \geqslant 0\right]$. Taking the vertex on the bottom parallelogram opposite $v_{3}$ as $v_{0}^{\prime}$, we see that $v_{1}^{\prime}=v_{4}$. Since the rim coordinates are $2\left(x_{1}+y_{2}, y_{1}-x_{2}\right)$, we compute the coordinates of $v_{0}^{\prime}$ as follows:

1. add $\frac{1}{2}$ the rim coordinates to the coordinates of $v_{3}$ to get $\left(x_{1}+y_{2}+x_{3}, y_{1}-x_{2}+y_{3}\right)$, the coordinates of the point on the bottom rim half way around the rim;

2. rotate this point $90^{\circ}$ clockwise about $v_{4}$ to get

$$
\left(y_{1}-x_{2}+y_{3}+x_{4}-y_{4},-x_{1}-y_{2}-x_{3}+x_{4}+y_{4}\right) \text {, the coordinates of } v_{0}^{\prime} \text {. }
$$

Since the two parallelograms for $\tau$ cannot be directly congruent, we must have $\left(x_{4}-\right.$ $\left.x_{3}, y_{4}-y_{3}\right) \neq\left(x_{2}, y_{2}\right)$. The coordinates of the unlabeled vertex on each parallelogram are then forced. Hence we have an eight parameter family: $\left\{x_{i}, y_{i}, i=1\right.$ to 4$\}$.

Groups (v) and (iv) have two reflections with planes for reflection intersecting in the axis of $\tau$. In these cases, the parallelogram of $\tau$ must admit two perpendicular reflections. We observe that if the axes of reflections are the diagonals of the parallelogram, they must both be color-preserving. We conclude that the parallelogram must be a rectangle with the axes of reflection perpendicular to the sides. Hence all sides have SSG coordinates of the form $(x)$ or all of the form $(x, x)$ or $(-x, x)$. In the first case, we again get only colorpreserving reflections. Hence $x_{1}=y_{1}=p$ and $-x_{2}=y_{2}=q$. The bottom parallelogram must also be a quadrilateral at a $45^{\circ}$ angle: $v_{4}-v_{3}=(s, s)$. In order for the centers of the sides to line up we must have $v_{3}+\left(\frac{s}{2}, \frac{s}{2}\right)=\left(\frac{p}{2}, \frac{p}{2}\right)+(r,-r)$. Solving the second equation for $v_{3}$ gives $v_{3}=\left(\frac{p-s}{2}+r, \frac{p-s}{2}-r\right)$ and then solving the first for $v_{4}$ gives $v_{4}=\left(\frac{p+s}{2}+r, \frac{p+s}{2}-r\right)$. The area of the top rectangle is $2 p q$, the area of the cylinder is $4 r(p+q)$ and the area of the bottom rectangle is $2 s(p+q-s)$. The formula for the number of vertices follows at once.

The groups (vi), (vii), (viii), and (ix) all have an equator of reflection perpendicular to the axis of $\tau$. In one case (viii), the reflection is color-preserving so the equator of reflection and the rim could be horizontal (however in this case, $y_{1}-x_{2}=0$ making $\tau$ as well as the reflection color-preserving) or it could be a color-preserving equator containing several degree- 3 vertices. With this one possible exception, the equator of reflection and the rim must be at a $45^{\circ}$ angle: $x_{1}+y_{2}=y_{1}-x_{2}$, giving $y_{2}=y_{1}-x_{1}-x_{2}$.

In the case of the color-preserving $45^{\circ}$ reflection $v_{3}$ has coordinates $(r,-r)$ with $r$ even; in the case of the color-reversing reflection $v_{3}$ has coordinates $(r,-r)$ with $r$ odd; in the case of the color-reversing rotatory-reflection $v_{3}$ has coordinates $\left(r+\frac{x_{1}+y_{2}}{2},-r+\frac{x_{1}+y_{2}}{2}\right)$ with $r$ odd. Since the bottom parallelogram is the reflected image of the top parallelogram, $x_{1}^{\prime}=$ $-x_{2}$ and $y_{1}^{\prime}=y_{1}$. This gives $x_{4}=x_{2}+x_{3}$ and $y_{4}=y_{3}-y_{2}$. Hence the only free parameters are $x_{1}, y_{1}, x_{2}$ and $x_{3}$. In terms of these parameters, the top cap, and hence the bottom cap too, has area $y_{1}^{2}-x_{1}^{2}-2 y_{1} x_{2}$. In both cases, the cylinder area is simply $4 r\left(y_{1}-x_{2}\right)$. Hence the number of vertices in the underlying $\mathrm{SG}$ is $\frac{1}{2}\left(y_{1}^{2}-x_{1}^{2}-2 * y_{1} x_{2}\right)+r\left(y_{1}-x_{2}\right)+1$. 


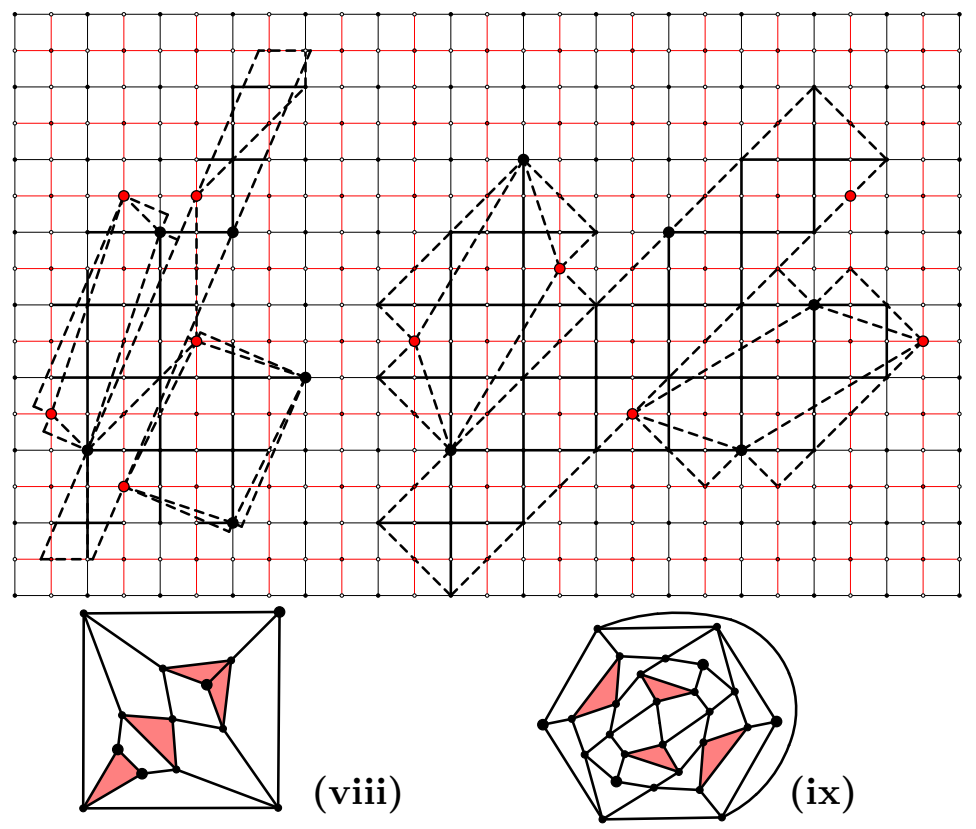

Figure 15: Smallest Examples (viii) \& (ix)

Since the SSGs with groups (iv) through (ix) have order 4, the corresponding SG has just one symmetry. When that symmetry is a half-turn or a reflection, the SG is drawn to exhibit that symmetry. However, in the case of (vii), the symmetry is the antipodal map $\alpha$ and we have labeled the vertices to identify this symmetry.

$\mathcal{A}_{\Gamma}$ is of Type (ix). Since the half-turn is color-preserving $x_{1}$ and $x_{2}$ have the same parity; since the rotatory-reflection is color-reversing, $x_{3}=r+\frac{x_{1}+y_{2}}{2}$ must be odd. The smallest example avoiding additional symmetries: $x_{1}=3, y_{1}=5, x_{2}=-1$ and $r=2$ for $\left|V_{b}\right|=26$.

$\mathcal{A}_{\Gamma}$ is of Type (viii). $\tau$ is color-reversing; the reflection is color-preserving. The smallest example involves an equator through four degree-3 vertices: $x_{1}=2, y_{1}=6,-x_{2}=y_{2}=1$, $x_{3}=-y_{3}=1$ and $x_{4}=y_{4}=3$ for $\left|V_{b}\right|=13$

$\mathcal{A}_{\Gamma}$ is of Type (vii). $\tau$ is color-reversing; the reflection is color-reversing. Smallest example $x_{1}=1, y_{1}=3, x_{2}=-2$ and $x_{3}=1$ for $\left|V_{b}\right|=16$.

$\mathcal{A}_{\Gamma}$ is of Type (vi). $\tau$ is color-preserving; the reflection is color-reversing. Smallest example $x_{1}=1, y_{1}=3, x_{2}=-3$ and $x_{3}=1$ for $\left|V_{b}\right|=20$.

With all of the constraints in Lemma 12 (ii) the next two smallest examples are easy to construct.

$\mathcal{A}_{\Gamma}$ is of Type (v). Smallest example $x_{1}=y_{1}=1,-x_{2}=y_{2}=4$. Then $x_{3}=4, y_{3}=0$, $x_{4}=1$ and $y_{4}=-3$ giving $\left|V_{b}\right|=16$.

$\mathcal{A}_{\Gamma}$ is of Type (iv). Smallest example $x_{1}=y_{1}=1,-x_{2}=y_{2}=5$. Then $x_{3}=4, y_{3}=0$, $x_{4}=1$ and $y_{4}=-3$ giving $\left|V_{b}\right|=20$. 

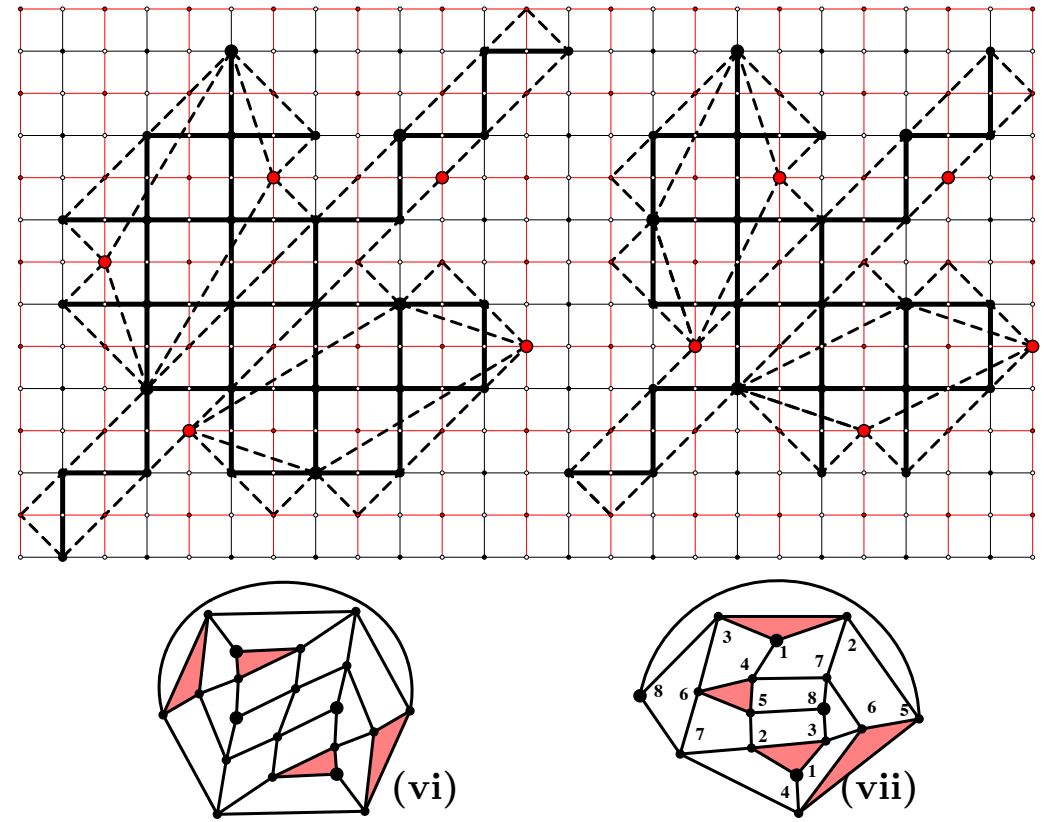

Figure 16: Smallest Examples (vi) \& (vii)

Finally, we come to the group (iii) consisting of one color-reversing half-turn and the identity. We must simply choose the parameters so that there are no additional symmetries.

$\mathcal{A}_{\Gamma}$ is of Type (iii). Smallest example $x_{1}=3, y_{1}=1, x_{2}=0, y_{2}=2, x_{3}=1, y_{3}=-1$, $x_{4}=0$ and $y_{4}=-2$ giving $\left|V_{b}\right|=8$.

Our final template covers just the remaining two groups: (i) a single color-reversing reflection and (ii) only the color-reversing antipodal map.

Basic Conditions: $y_{1} \geqslant x_{1}, x_{1} y_{3}-x_{3} y_{1} \geqslant 0, y_{3} \geqslant-x_{3}, x_{2}=y_{1}-y_{3}$; $y_{2} \leqslant y_{1}+y_{3}, \quad v_{4}=(p,-p)$ with $p$ odd, for (i);

$\left(p+\frac{x_{1}+y_{2}-x_{3}}{2},-p+\frac{x_{1}+y_{2}-x_{3}}{2}\right)$ with $p+\frac{x_{1}+y_{2}-x_{3}}{2}$ odd, for (ii).

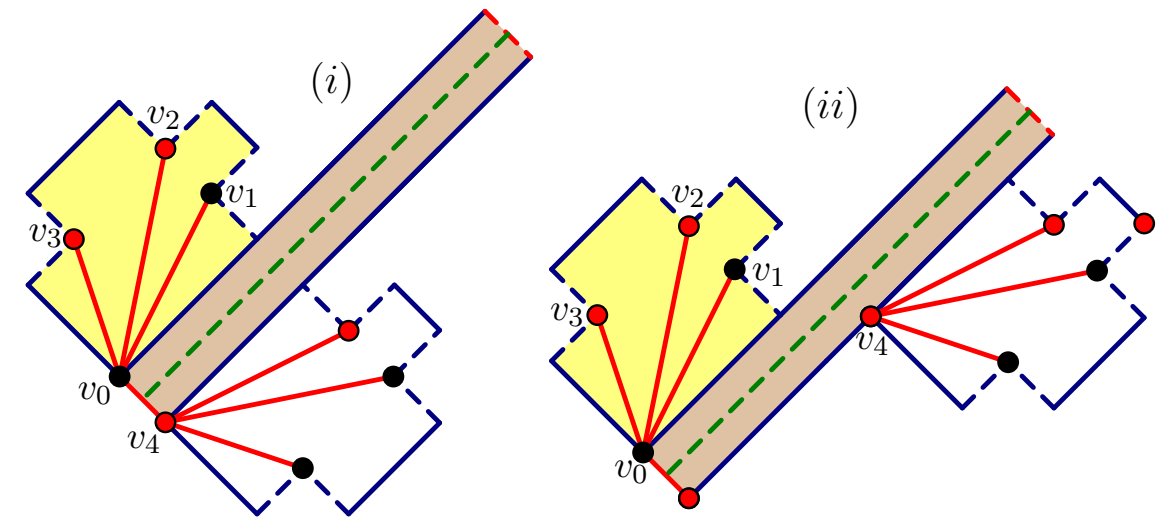

Template \#4: One color-reversing opposite symmetry.

Lemma 13. Let $\Gamma$ be an $S S G$ that admits only one symmetry: either a color-reversing reflection or the color-reversing antipodal map. Then it may be orientated as in Template 


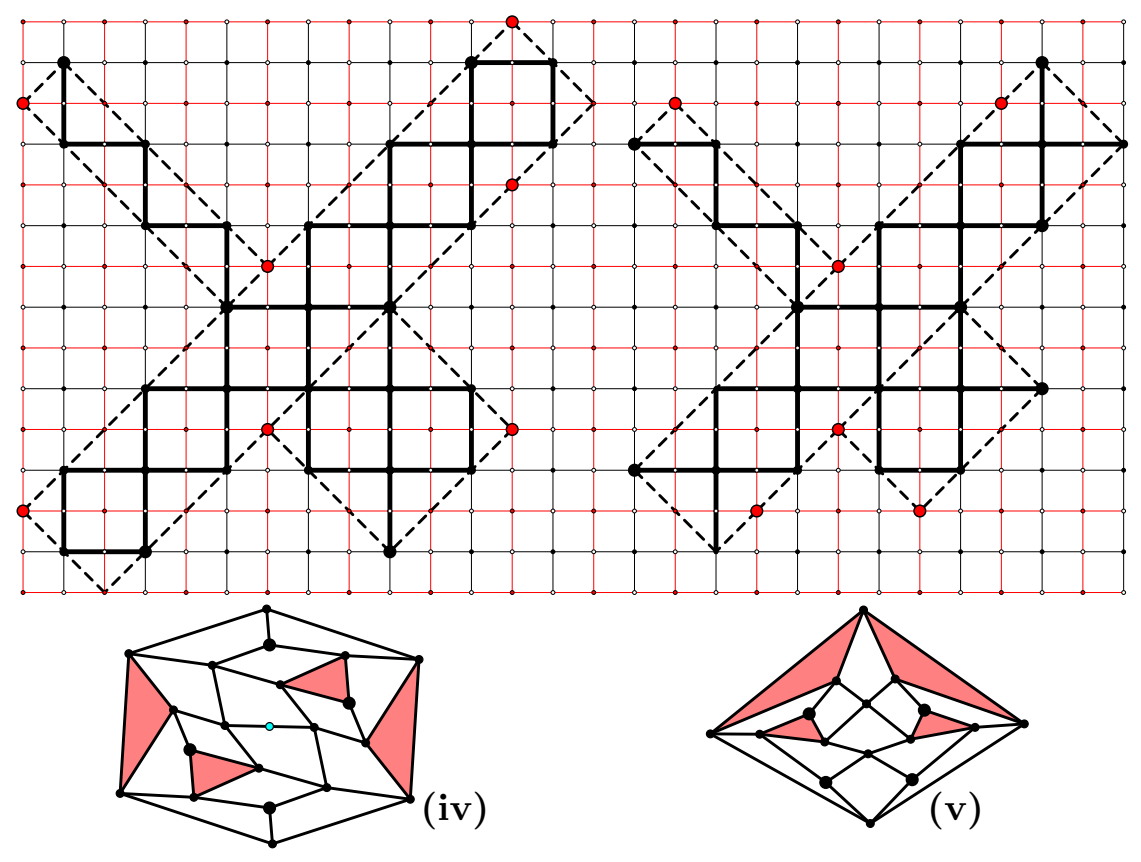

Figure 17: Smallest Examples (iv) \& (v)

\#4 and the basic conditions listed must be satisfied. Furthermore in both cases

$$
\left|V_{b}\right|=y_{1} y_{3}-\frac{\left(y_{1}-x_{1}\right)^{2}+\left(y_{3}+x_{3}\right)^{2}+\left(y_{1}+y_{3}-y_{2}\right)^{2}}{4}+\frac{p\left(x_{1}+y_{2}-x_{3}\right)}{2}
$$
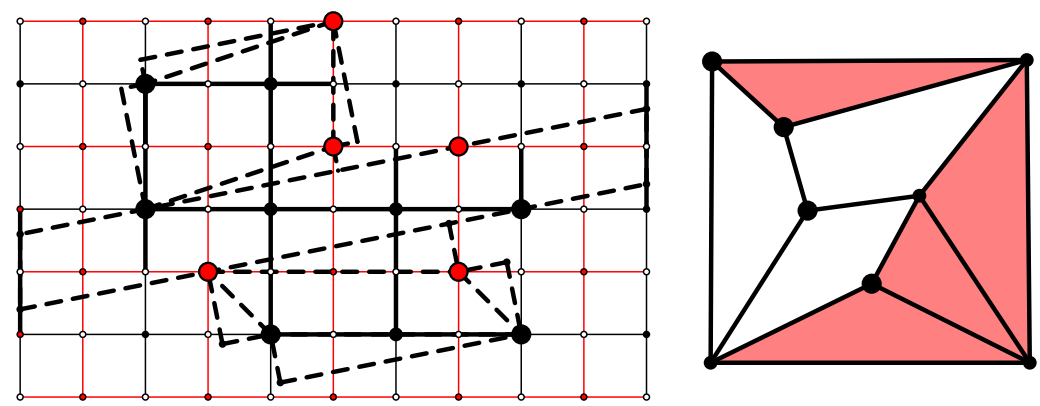

Figure 18: Smallest Example (iii)

Proof. Suppose that $\mathcal{A}_{\Gamma}$ is of Type (i). Then four of the degree-3 vertices are on one side of (above) the axis of the reflection with the other four on the other side (below) the axis. Let $v_{0}$ be the degree- 3 vertex above the axis closest to the axis. Then the $45^{\circ}$ through $v_{0}$ and parallel to the axis of reflection must be the top rim. If $\mathcal{A}_{\Gamma}$ is of Type (ii), let $u_{1}$ and $u_{2}$ be nearest neighbors among all pairs of degree- 3 vertices. Among the four vertices of degree-3 different from $u_{1}, u_{2}, \alpha\left(u_{1}\right)$ and $\alpha\left(u_{2}\right)$, let $u_{3}$ be the nearest to one of $u_{1}$ and $u_{2}$; then choose $u_{4}$ to be the degree- 3 vertex different from $u_{1}, u_{2}, u_{3}, \alpha\left(u_{1}\right), \alpha\left(u_{2}\right)$ and $\alpha\left(u_{3}\right)$. that is closest to the convex hull of $u_{1}, u_{2}$ and $u_{3}$. Let $(p, q)$ denote the rim coordinates of 
the quadrilateral $u_{1}, u_{2}, u_{3}$ and $u_{4}$. Since the quadrilateral $\alpha\left(u_{1}\right), \alpha\left(u_{2}\right), \alpha\left(u_{3}\right)$ and $\alpha\left(u_{4}\right)$ is the reflected image of the quadrilateral $u_{1}, u_{2}, u_{3}$ and $u_{4}$ its rim coordinates are $(q, p)$. But the two caps must have the same coordinates, hence $p=q$ and the rims are $45^{\circ}$ lines. Now, if $u_{1}, u_{2}, u_{3}$ and $u_{4}$ are relabeled $v_{0}, v_{1}, v_{2}$ and $v_{3}$ in counterclockwise order with $v_{0}$ on the rim, note that $v_{4}=\alpha\left(v_{0}\right)$ is half way around on the other rim. We now have the diagram on the right in the template. The basic conditions then follow directly from Lemma 8.
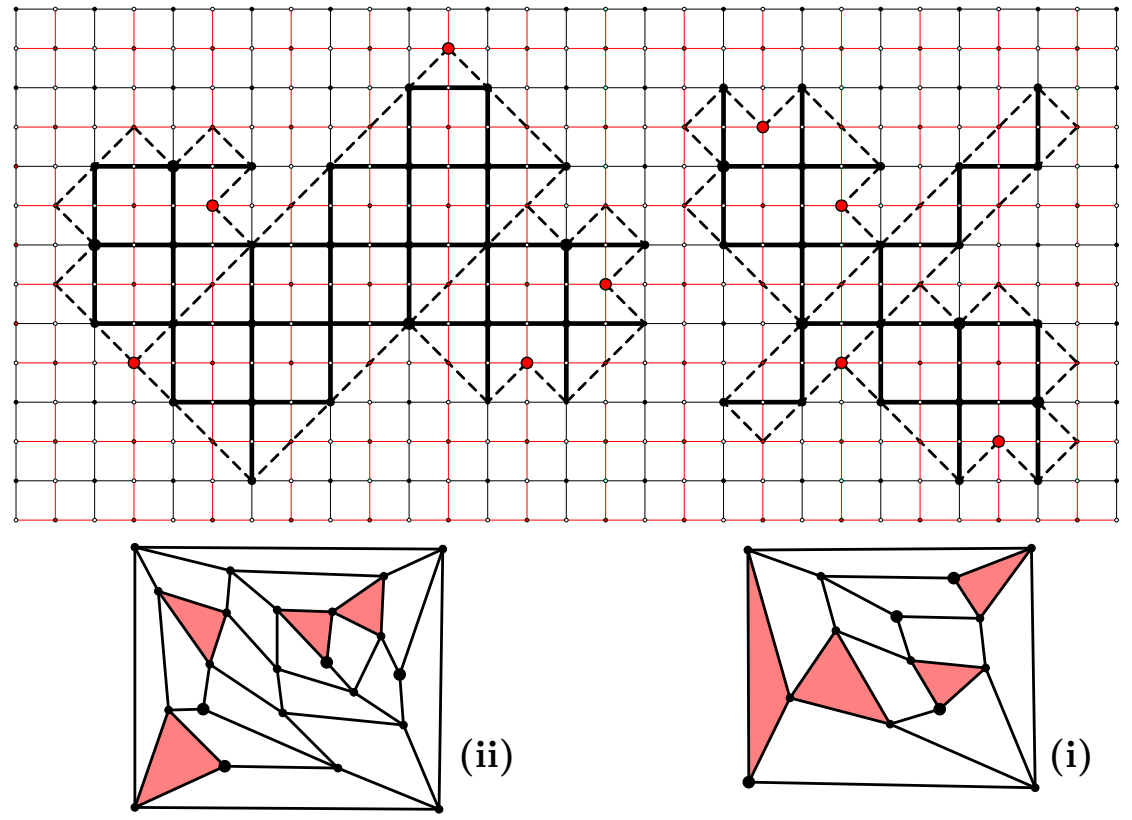

(i)

Figure 19: Smallest Examples (ii) \& (i)

It should be noted that unlike the previous three templates, the basic conditions here do not exclude the possibility of additional symmetries. Hence care must be taken when using this template to check that the resulting SG admits no reflection or half-turn. Also, a Type (ii) SSG could admit several decompositions and so in this case two different choices for the parameter values could result in the same SSG.

$\mathcal{A}_{\Gamma}$ is of Type (ii). Smallest example $x_{1}=2, y_{1}=4, x_{2}=1, y_{2}=5$. Then $x_{3}=-1$, $y_{3}=3, x_{4}=7$ and $y_{4}=1$ giving $\left|V_{b}\right|=22$.

$\mathcal{A}_{\Gamma}$ is of Type (i). Smallest example $x_{1}=1, y_{1}=3, x_{2}=-1, y_{2}=5$. Then $x_{3}=-2$, $y_{3}=4, x_{4}=1$ and $y_{4}=-1$ giving $\left|V_{b}\right|=14$.

\section{References}

[1] D. Archdeacon, R. B. Richter, Construction and classification of self-dual spherical polyhedra, J. Comb. Theo. B 54, 37-63 (1992). 
[2] G. Brinkmann, S. Greenberg, C. Greenhill, B. McKay, R. Thomas and P. Wollan, Generation of simple quadrangulations of the sphere, Disc. Math. 305, 33-54, (2005).

[3] H. M. S. Coxeter and W. O. Moser, Generators and Relations for Discrete Groups, Springer, Berlin 1957.

[4] M. Dutour, M. Deza, Goldberg-Coxeter construction for 3- and 4-valent plane graphs, Electr. J. Comb. 11 No.1, (2004).

[5] B. Servatius and P. R. Christopher, Construction of self-dual graphs, Amer. Math. Monthly 99 No.2, 153-158, (1992).

[6] B. Servatius, H. Servatius, Self-dual maps on the sphere, Disc. Math. 134, 139-150, (1992).

[7] B. Servatius and H. Servatius, The 24 symmetry pairings of self-dual maps on the sphere, Disc. Math. 140, 167-183, (1995).

[8] B. Servatius, H. Servatius, Self-dual graphs, Disc. Math. 149, 223-232, (1996).

[9] C. A. B Smith and W. T. Tutte, A class of self-dual maps, Cand. J. Math., 2, 179-196 (1950). 